\title{
Stimulation of Baroresponsive Parts of the Nucleus of the Solitary Tract Produces Nitric Oxide-mediated Choroidal Vasodilation in Rat Eye
}

\author{
Chunyan $L^{1}{ }^{1}$, Malinda E. C. Fitzgerald ${ }^{1,2,3}$, Nobel Del Mar ${ }^{1}$ and Anton Reiner ${ }^{1,2 *}$ \\ ${ }^{1}$ Department of Anatomy and Neurobiology, The University of Tennessee Health Science Center, Memphis, TN, USA, \\ ${ }^{2}$ Department of Ophthalmology, The University of Tennessee Health Science Center, Memphis, TN, USA, ${ }^{3}$ Department \\ of Biology, Christian Brothers University, Memphis, TN, USA
}

Preganglionic parasympathetic neurons of the ventromedial part of the superior salivatory nucleus (SSN) mediate vasodilation of orbital and choroidal blood vessels, via their projection to the nitrergic pterygopalatine ganglion (PPG) neurons that innervate these vessels. We recently showed that the baroresponsive part of the nucleus of the solitary tract (NTS) innervates choroidal control parasympathetic preganglionic neurons of SSN in rats. As this projection provides a means by which blood pressure (BP) signals may modulate choroidal blood flow (ChBF), we investigated if activation of baroresponsive NTS evokes ChBF increases in rat eye, using Laser Doppler Flowmetry (LDF) to measure ChBF transclerally. We found that electrical activation of ipsilateral baroresponsive NTS and its efferent fiber pathway to choroidal SSN increased mean ChBF by about $40-80 \%$ above baseline, depending on current level. The ChBF responses obtained with stimulation of baroresponsive NTS were driven by increases in both choroidal blood volume (ChBVol; i.e., vasodilation) and choroidal blood velocity

Edited by:

Jose L. Lanciego,

University of Navarra, Spain

Reviewed by:

Jeffrey Kiel,

University of Texas Health Science

Center at San Antonio, USA

Leopold Schmetterer,

Medical University of Vienna, Austria

*Correspondence:

Anton Reiner

areiner@uthsc.edu

Received: 05 August 2016 Accepted: 21 September 2016

Published: 07 October 2016

Citation:

Li C, Fitzgerald MEC, Del Mar N and

Reiner A (2016) Stimulation of Baroresponsive Parts of the Nucleus of the Solitary Tract Produces Nitric

Oxide-mediated Choroidal

Vasodilation in Rat Eye.

Front. Neuroanat. 10:94.

doi: 10.3389/fnana.2016.00094 (ChBVel; possibly due to orbital vessel dilation). Stimulation of baroresponsive NTS, by contrast, yielded no significant mean increases in systemic arterial blood pressure (ABP). We further found that the increases in ChBF with NTS stimulation were significantly reduced by administration of the neuronal nitric oxide (NO) synthase inhibitor $\mathrm{N}^{\omega}$-propylI-arginine (NPA), thus implicating nitrergic PPG terminals in the NTS-elicited ChBF increases. Our results show that the NTS neurons projecting to choroidal SSN do mediate increase in ChBF, and thus suggest a role of baroresponsive NTS in the BP-dependent regulation of ChBF.

Keywords: choroidal blood flow, superior salivatory nucleus, nucleus of solitary tract, autonomic, parasympathetic

\footnotetext{
Abbreviations: ABP, arterial blood pressure; AP, area postrema; BP, blood pressure; C, central subnucleus of NTS; ChBF, choroidal blood flow; ChBVol, choroidal blood volume; ChBVel, choroidal blood velocity; cSSN, choroidal subdivision of superior salivatory nucleus; Com, commissural nucleus of NTS; Cu, cuneate nucleus; CVLM, caudal ventrolateral medulla; $\mathrm{D}$, dorsal nucleus of NTS; DAB, diaminobenzidine tetrahydrochloride; eNOS, endothelial nitric oxide synthase; G, gelatinous subnucleus of NTS; Gr, gracile nucleus; HR, heart rate; iM, intermediate subnucleus of NTS; M, medial subnucleus of NTS; MLF, Medial longitudinal fasciculus; nNOS, neuronal nitric oxide synthase; NO, nitric oxide; NPA, $\mathrm{N}^{\omega}$-propyl-1-arginine; NTS, nucleus of solitary tract; Ox, blood oxygenation; Pr, nucleus prepositus; PPG, pterygopalatine ganglion; RVLM, rostral ventrolateral nucleus of medulla; sol, solitary tract and subnucleus of NTS; SSN, superior salivatory nucleus; stim, stimulation; V, ventral subnucleus of NTS; VeM, medial vestibular nucleus; VeS, superior vestibular nucleus; VIP, vasoactive intestinal polypeptide; VL, ventrolateral subnucleus of NTS; 10, dorsal vagal motor nucleus; 12, hypoglossal motor nucleus.
} 


\section{INTRODUCTION}

The retina has two separate vascular supplies involved in its support: the retinal vessels within the retina itself, and the choroidal vessels external to Bruch's membrane and the retinal pigment epithelium (Alm, 1992). The retinal vessels provide blood supply to the inner two-thirds of the retina, while the outer retina is avascular and nourished from the choroid (Alm, 1992). The choroid provides $65-85 \%$ of the oxygen to the retina, with variation among species (Alm and Bill, 1973; Alm, 1992). Diminishing choroidal blood flow (ChBF) by occluding one or more the posterior ciliary arteries feeding choroid (Loeffler et al., 1994; Hayreh, 2004) or occluding a vortex vein (Collier, 1967), rapidly destroys the outer retina, emphasizing the importance of $\mathrm{ChBF}$ for outer retinal health. Moreover, reductions in $\mathrm{ChBF}$ are observed in aging and various retinal diseases, such as systemic hypertension, diabetic retinopathy and age-related macular degeneration (Grunwald et al., 1998a,b, 2005; Fitzgerald et al., 2001; Ito et al., 2001; Pournaras et al., 2006; Metelitsina et al., 2008), raising the possibility that the ChBF reductions contribute to the retinal declines observed.

Although ChBF was once thought to respond passively to changes in perfusion pressure, recent studies have shown that ChBF remains stable over a systemic blood pressure (BP) range of about 35\% above and below basal (Kiel and Shepherd, 1992; Lovasik et al., 2003; Reiner et al., 2003, 2011), with neural mechanisms apparently involved in the compensation (Kiel, 1999; Li et al., 2010, 2015; Reiner et al., 2010b). When BP is low, choroidal blood vessels dilate to maintain blood flow to the eye, and when BP is high, choroidal blood vessels constrict, preventing overperfusion, tissue edema, and oxidative injury. Neurogenic choroidal vasodilation is mediated by parasympathetic and sensory innervation of choroidal vessels, and neurogenic choroidal vasoconstriction is accomplished by sympathetic innervation. Some parts of the central circuitry subserving parasympathetic contributions to choroidal baroregulation have been characterized. In birds and mammals both, the parasympathetic pterygopalatine ganglion (PPG) innervates orbital vessels feeding the choroid, as well as choroidal vessels themselves (Stone et al., 1987; Cuthbertson et al., 1997, 2003; Jablonski et al., 2007; Reiner et al., 2012). In mammals, the choroid is supplied by the posterior and anterior ciliary arteries, arising from the ophthalmic artery, while in birds the choroidal arteries arising from the ophthalmotemporal artery supply the choroid (Cuthbertson et al., 1997; Morrison et al., 1999; Reiner et al., 2012). The PPG input to choroid and the choroidal feeder vessels employ the vasodilators nitric oxide (NO) and vasoactive intestinal polypeptide (VIP), and as a result the ophthalmic and posterior ciliary arteries in mammals and the choroidal and ophthalmotemporal arteries in birds, as well as choroidal vessels, are densely coated with nerve fibers containing neuronal NO synthase (which synthesizes NO in neurons) and VIP (Stone et al., 1987; Cuthbertson et al., 1997; Reiner et al., 2012). The preganglionic neurons regulating the PPG reside in the superior salivatory nucleus (SSN), the autonomic component of the facial motor complex in the hindbrain
(Cuthbertson et al., 2003). In prior studies on the inputs to the SSN in rats, we found that a major input arose from the nucleus of the solitary tract (NTS) of the medulla (Li et al., 2010), and in more recent studies we have characterized the part of NTS from which this input arises (Li et al., 2015).

The NTS is organized into viscerotopic subdivisions that receive input selectively from the tongue, vasculature and thoracic and abdominal viscera (Loewy, 1990; Paton, 1999; Pilowsky and Goodchild, 2002). Cardiovascular afferents predominantly project to the ipsilateral interstitial and dorsal NTS subnuclei at and anterior to the level of the obex (Ciriello, 1983; Zhang and Ashwell, 2001). Our studies have demonstrated that the part of NTS receiving baroreceptor information lies within the region of NTS that projects directly to the ChBFcontrol neurons of SSN (Li et al., 2015). Since NTS input to SSN is excitatory (Agassandian et al., 2002, 2003; Li et al., 2015) and SSN activation increases ChBF (Steinle et al., 2000; Fitzgerald et al. unpublished observations), it seems likely that the NTS also exerts a vasodilatory effect on choroid. The present study directly examined the influence of NTS on the ipsilateral ChBF using Laser Doppler Flowmetry (LDF). We found that activation of the parts of NTS receiving baroreceptive input and/or responsive to BP signals increases ipsilateral $\mathrm{ChBF}$, and that the peripheral PPG terminals mediating this effect appear to use NO as a vasodilator. These results are consistent with the view that the NTS is part of the circuit for the adaptive parasympathetic regulation of ChBF during systemic hypotension.

\section{MATERIALS AND METHODS}

\section{Experimental Overview}

Adult male Sprague-Dawley (SD) rats (290-380 g; from Harlan, Indianapolis, IN, USA) were used in all present studies. Our overall goal was to identify the sites within NTS from which ChBF increases could be elicited, and relate them to the location of the subdivisions within NTS that are baroresponsive and project to prechoroidal SSN. To this end, we mapped the distribution within NTS of neurons that express c-fos upon systemic hypotension, and related this to the published terminal field within NTS of aortic and carotid sinus baroreceptor afferent input via the vagus and glossopharyngeal nerves (Ciriello, 1983; Housley et al., 1987; Altschuler et al., 1989), the distribution of neurons within NTS that project to prechoroidal SSN identified in our own prior work ( $\mathrm{Li}$ et al., 2015), our own NTS stimulation mapping, and the published distribution of BP-responsive NTS sites (Rogers et al., 1993; Mayne et al., 1998). We used the systematic sampling of NTS sites with electrical stimulation while monitoring $\mathrm{ChBF}$ and systemic $\mathrm{BP}$ to map ChBF-responsive NTS sites. Histological analysis was used to reconstruct the stimulation sites. Additionally, in a separate set of rats, we examined the effect of neuronal nitric oxide synthase (nNOS) inhibition on ChBF responses to activation of NTS sites yielding $\mathrm{ChBF}$ increases, to assess the involvement of peripheral NO-mediated mechanisms in the parasympathetic choroidal vasodilation observed with 
activation of effective NTS sites. All experiments were in compliance with the ARVO statement on the Use of Animals in Ophthalmic and Vision Research, and with NIH and institutional guidelines.

\section{Systemic Hypotension and NTS Responses} Twenty-two adult male SD rats (280-440 g) were anesthetized for 90-150 min, using ip injection of $0.1 \mathrm{ml} / 100 \mathrm{~g}$ of a ketamine/xylazine mixture $(87 / 13 \mathrm{mg} / \mathrm{kg})$, with supplemental doses (0.05-0.07 $\mathrm{ml}$ of ketamine/xylazine mixture) every 25-30 min. The right femoral artery was cannulated for systemic arterial blood pressure (ABP) recording, and the right femoral vein for blood withdrawal. Body temperature was maintained at $37^{\circ} \mathrm{C}$ with a Harvard heating blanket and rectal thermoprobe. To prepare the surgical site, hair was removed by clippers from the ventrum of the hind legs. To create hypotension, 4-7 $\mathrm{ml}$ of blood was slowly withdrawn via the cannula in the right femoral vein. The hypotensive state was maintained for 40 to $160 \mathrm{~min}$. Control rats were similarly prepared, but no blood withdrawn. After surgery, the rats were perfused with $4 \%$ paraformaldehyde prepared in $0.1 \mathrm{M}$ sodium phosphate buffer (PB; $\mathrm{pH} 7.4$ ) with $0.1 \mathrm{M}$ lysine and $0.01 \mathrm{M}$ sodium periodate (PLP fixative). The brains were removed, cryoprotected at $4^{\circ} \mathrm{C}$, and sectioned frozen at $40 \mu \mathrm{m}$ on a sliding microtome into eight separate series for each brain. Selected series of sections were incubated in rabbit-anti-c-fos diluted at 1:1000 (sc-52; Santa Cruz Biotechnology; Santa Cruz, CA, USA) overnight at room temperature. After rinsing, the sections were incubated in donkey-anti rabbit secondary antibody (1:200; Jackson ImmunoResearch Laboratories, Inc., West Grove, PA, USA) for $1 \mathrm{~h}$ and rabbit peroxidase-anti-peroxidase (Rb PAP; 1:1000; Jackson ImmunoResearch Laboratories, Inc., West Grove, PA, USA) for $1 \mathrm{~h}$. The c-fos labeling was visualized using diaminobenzidine tetrahydrochloride (DAB), using a standard brown DAB reaction as in our prior studies (Li et al., 2010, 2015). The sections were then rinsed, mounted on gelatin-coated slides, air-dried, dehydrated and coverslipped with Permount ${ }^{\circledR}$ (Fisher Scientific, Pittsburgh, PA, USA).

\section{Choroidal Blood Flow Surgical Preparation}

Prior to surgery, 24 rats were anesthetized by ip injection of $0.1 \mathrm{ml} / 100 \mathrm{~g}$ of a ketamine/xylazine mixture $(87 / 13 \mathrm{mg} / \mathrm{kg})$, with supplemental doses (0.05-0.07 $\mathrm{ml}$ of ketamine/xylazine mixture) every 25-30 min. To prepare the surgical sites, hair was removed by clippers from the dorsum of the head and ventrum of the hind legs. Body temperature was maintained at $37^{\circ} \mathrm{C}$ with a Harvard heating blanket and rectal thermoprobe. To measure systemic $\mathrm{ABP}$, the femoral artery was catheterized as follows. Segments of PE-50 polyethylene tubing that were $20-30 \mathrm{~cm}$ long were pre-soaked with heparinized saline (40 units $/ \mathrm{ml}$ ) for $24 \mathrm{~h}$ to prevent catheter-induced clot formation. Both femoral artery and vein were isolated from the surrounding connective tissue and cannulated using such tubing. A Digi-Med Blood Pressure Analyzer $^{\mathrm{TM}}$ (BPA-100, Micro-Med, Inc., Louisville, KY, USA) was used to measure $A B P$ and heart rate $(\mathrm{HR})$ via a pressure transducer connected to the femoral artery cannula. The femoral vein was cannulated for administration of $\mathrm{N}^{\omega}$-propyl-l-arginine (NPA, 1-2 mg/kg, Tocris Bioscience), a highly selective NO synthase 1 (NOS1, i.e., neuronal NOS) inhibitor (Zhang et al., 1997; Cooper et al., 2000). The rats were then positioned in a stereotaxic device. A pulse oximeter on the tail was used to measure systemic blood oxygen saturation.

To prepare the eye for transcleral measurement of ChBF, fascia overlying the superior pole of the right eye and the Harderian gland were removed. The tip of a laser Doppler probe connected to a LASERFLO BPM ${ }^{2}$ blood perfusion monitor (Vasamedics; Eden Prairie, MN, USA or BPM 403A; TSI Incorporated., St. Paul, MN, USA) was positioned 1-3 mm above the sclera with a micromanipulator. Measurements of $\mathrm{ChBF}$ were made from the vascular bed beneath the sclera in the gap between the superior and medial rectus muscles, at the equator of the superior part of the eye. A small amount of Aquasonic ultrasound gel (Parker Laboratories, Inc., Fairfield, NJ, USA) or $33 \%$ glycerol- $0.1 \mathrm{M}$ sodium $\mathrm{PB}$, was used in the interface between the probe tip and the sclera to prevent tissue drying during the experiment. For placement of a stimulating electrode, the skin over the skull was retracted and bone over NTS removed. The coordinates for NTS from the rat brain atlas of Paxinos and Watson (2009) were: - $13.6 \mathrm{~mm}$ behind Bregma, -1.0 lateral to Bregma, and -5.2 below the brain surface. An insulated, stainless steel stimulating electrode (5M $\Omega$; Catlog \# 572000; A-M Systems Inc., Sequim, WA, USA) was then positioned stereotaxically in the right NTS. To minimize the damage to NTS during penetration, we also used glass micropipette electrodes for seven rats. Micropipettes were pulled from glass tubing (World Precision Instrument, 1B150F-4) with a PE-2 microelectrode puller (Narashige), and micropipettes with a diameter of 50-100 $\mu \mathrm{m}$ used for stimulation. Micropipettes were filled with artificial cerebral spinal fluid (ACSF) composed of (in $\mathrm{mM}$ ): $126 \mathrm{NaCl}, 26$ $\mathrm{NaHCO}_{3}, 3 \mathrm{KCl}, 1.25 \mathrm{NaH}_{2} \mathrm{PO}_{4}, 2 \mathrm{MgCl}_{2}, 2 \mathrm{CaCl}_{2}$, and 20 glucose; $\mathrm{pH}$ 7.3, $310 \mathrm{mOsm}$, and a platinum wire was inserted.

\section{Choroidal Blood Flow Measurement}

$\mathrm{ChBF}$ was measured in the right eye using a laser Doppler flow probe connected to a LASERFLO $\mathrm{BPM}^{2}$ blood perfusion monitor. Our previous work has shown that ChBF can reliably be measured transclerally, using LDF (Fitzgerald et al., 1990, 1996, 2001, 2005; Zagvazdin et al., 1996b; Reiner et al., 2010b). The principles of LDF have been described in detail by others (Haumschild, 1986; Bonner and Nossal, 1990), and this approach provides an accepted relative index of $\mathrm{ChBF}$, as well as relative indices of choroidal blood volume (ChBVol) and choroidal blood velocity ( $\mathrm{ChBVel}$ ), whose product is $\mathrm{ChBF}$ and which have been shown to linearly relate to $\mathrm{ChBF}$ as measured by LDF (Riva et al., 1994; Shepherd et al., 1997; Grunwald et al., 1998a; Chou et al., 2002; Gugleta et al., 2002; Garhöfer et al., 2005; Tonini et al., 2010; Xu et al., 2010; Falsini et al., 2011). Moreover, hemodilution studies in model systems have shown that blood flow as assessed by laser Doppler scales linearly with red blood cell count (Nilsson et al., 1980; Fischer et al., 1985; Almond and Wheatley, 1992). 
Nonetheless, studies comparing $\mathrm{ChBVol}$ and $\mathrm{ChBVel}$ values determined by LDF with choroidal red blood cell concentration and velocity determined by other means would be useful for better understanding the choroidal vascular dynamics suggested by LDF data. Although the LASERFLO BPM ${ }^{2}$ nominally presents ChBF values in millilitre blood per minute per $100 \mathrm{~g}$ tissue, ChBVol in 12,000 red blood cells per cubic millimeter of tissue, and $\mathrm{ChBVel}$ as $10 \mathrm{~mm} / \mathrm{s}$, because of uncertainties about how well choroidal tissue matches the assumptions of the algorithms used to derive these measures, $\mathrm{ChBF}$ is reported here as relative blood flow units (BFUs), ChBVol as relative blood volume units (BVolU), and $\mathrm{ChBVel}$ as relative blood velocity units (BVelU). The output of our LASERFLO BPM ${ }^{2}$ was imported to a ML880 PowerLab 16/30 data acquisition system (ADInstruments Inc., Colorado Springs, CO, USA), and the digitized data were collected and analyzed using LabChart v7 Pro software (ADInstruments Inc., Colorado Springs, CO, USA) installed on an iMac Apple computer. The data averaging window of the $\mathrm{BPM}^{2}$ was set at $0.3 \mathrm{~s}$, with the sampling rate on PowerLab set at 200 samples/s.

\section{Choroidal Blood Flow: Stimulation of NTS}

Microstimulation consisted of a $7 \mathrm{~s}$ train of $100 \mathrm{~Hz}, 0.5 \mathrm{~ms}$ duration anodal or cathodal current pulses delivered by a $S 48$ stimulator (Grass Instrument Company, Quincy, MA, USA) and PSIU6 stimulus isolation unit (Grass Instrument Company, Quincy, MA, USA). The amplitude of the anodal current pulses was varied between $50-100 \mu \mathrm{A}$, while for cathodal current pulses were $20 \mu \mathrm{A}$. For these rats, we systematically sampled NTS sites at a series of penetrations that encompassed the rostralcaudal, medial-lateral, and dorsal-ventral extent of NTS. In our initial studies $(n=17)$, we used anodal stimulation as we had in prior ChBF studies (Fitzgerald et al., 1990, 1996, 2005). Because axons within $300-500 \mu \mathrm{m}$ of the electrode tip are more readily stimulated than neuronal cell bodies by $50-100 \mu \mathrm{A}$ anodal current pulses of $0.5 \mathrm{~ms}$ duration (Bagshaw and Evans, 1976), we found that anodal stimulation tended to identify the course taken by NTS axons en route to prechoroidal SSN, especially at the lower current levels. To better define the location of the perikarya within NTS driving ChBF increases, we used cathodal stimulation of NTS in systematic mapping studies in a second set of rats $(n=7)$, because it more effectively stimulates the axon hillock of perikarya (Ranck, 1975; Merrill et al., 2005; Lu et al., 2008), thereby providing better localization of the perikarya that drive ChBF increases from within NTS. Data presented here for each rat represent an average of 3-7 NTS stimulation trials. Data on $\mathrm{ChBF}, \mathrm{ChVol}$, ChBVel, and ABP are presented. For ChBF, $\mathrm{ChVol}$, and ChVel, data are presented in arbitrary units, as in our prior studies (Fitzgerald et al., 2001, 2005; Reiner et al., 2003, 2010b, 2011).

\section{Inhibition of $\mathbf{n N O S}$}

In a separate set of eight rats prepared as described above for ChBF and ABP measurement and NTS stimulation, we studied the effects of nNOS inhibition on the ChBF increasing effects of NTS stimulation. In these studies, electrodes were placed in the right NTS, as confirmed by the evoked ChBF increases and subsequent histological evaluation of electrode placement. The effects of $100 \mu \mathrm{A}$ current pulses on ChBF was determined prior to nNOS inhibition, in 3-7 stimulation trials. We then administered NPA (1-2 mg/kg, Tocris Bioscience) iv, and examined the effect on baseline ChBF and NTS-evoked increases in ChBF, ChBVol, ChBVel and arterial BP. After NPA administration, an additional 3-7 NTS stimulation trials were performed for these eight rats.

\section{Choroidal Blood Flow-Histological Analysis of Stimulation Sites}

At the end of each ChBF recording session, rats were perfused transcardially with $150-200 \mathrm{ml}$ sodium phosphate buffered saline (PBS, $0.85 \%$ sodium chloride dissolved in $0.01 \mathrm{M} \mathrm{PB}$ ), followed by $400-500 \mathrm{ml}$ fixative of $4 \%$ paraformaldehyde prepared in $0.1 \mathrm{M}$ sodium $\mathrm{PB}(\mathrm{pH} 7.4)$ with $0.1 \mathrm{M}$ lysine and $0.01 \mathrm{M}$ sodium periodate (the PLP fixative). Brains were removed and cryoprotected at $4^{\circ} \mathrm{C}$ for at least $24 \mathrm{~h}$ in $20 \%$ sucrose $-10 \%$ glycerol $-0.138 \%$ sodium azide in $0.1 \mathrm{M}$ sodium $\mathrm{PB}$. Brains were then frozen with dry ice, and coronal sections cut with a sliding microtome at $40 \mu \mathrm{m}$. Sections were collected as six parallel series. The free-floating sections were stored at $4^{\circ} \mathrm{C}$ in a $0.02 \%$ sodium azide $/ 0.02 \%$ imidazole in $0.1 \mathrm{M} \mathrm{PB}$ solution until staining procedures were carried out.

We used three histological methods to confirm that the electrode placement was within NTS and led to NTS activation: (1) analysis of the location of the electrode tip in NTS in cresyl violet-stained sections; (2) analysis of NTS activation in c-fos immunostained sections; and (3) analysis of NTS activation in sections immunolabeled for both parvalbumin and c-fos. While approaches 1 and 2 were effective in most cases, the paucity of parvalbumin in NTS (allowing its use as a negative marker of NTS) made it possible to unambiguously ascertain whether the electrode tip was in NTS and whether cfos-labeled neurons were in NTS. Our immunolabeling and cresyl violet staining procedures have been described in detail in our previous publications (Deng et al., 2007; Li et al., 2010, 2015). For the present two-color double labeling, sections were incubated in a rabbit primary antibody against $c$-fos at a 1:500 dilution (sc-52; Santa Cruz Biotechnology, Santa Cruz, CA, USA) overnight. After rinsing, the sections were incubated in donkey-anti rabbit secondary antibody (1:200; Jackson ImmunoResearch Laboratories, Inc., West Grove, PA, USA) for $1 \mathrm{~h}$ and rabbit peroxidase-anti-peroxidase (rabbit PAP; 1:1000; Jackson ImmunoResearch Laboratories, West Grove, PA, USA) for $1 \mathrm{~h}$. Then, the $\mathrm{c}$-fos signals were visualized by nickel-intensified $\mathrm{DAB}$ staining, yielding black nuclear labeling of c-fos+ neurons. Subsequently, the sections were incubated overnight in a mouse primary antibody against parvalbumin (1:5000; Sigma; P-3171). After rinsing, the sections were incubated in a donkey-anti-mouse secondary antibody and mouse PAP. Then the sections were processed using a standard brown $\mathrm{DAB}$ reaction, yielding brown parvalbumin neurons and processes. The $\mathrm{H}_{2} \mathrm{O}_{2}$ incubation time for the brown $\mathrm{DAB}$ reaction was $2 \mathrm{~min}$ (rather than the standard 
$10 \mathrm{~min}$ ) so that the parvalbumin immunolabeling would be light and not interfere with detection of c-fos+ neurons. The sections were then rinsed, mounted on gelatin-coated slides, air-dried, dehydrated and coverslipped with Permount ${ }^{\circledR}$ (Fisher Scientific, Pittsburgh, PA, USA). The sections were examined with a Olympus $\mathrm{BH}-2$ microscope with standard transmitted light. Images were captured using a Spot Idea ${ }^{\mathrm{TM}}$ camera and a Spot imaging software (Diagnostic Instruments, Inc., Sterling Heights, MI, USA). Note that only data from rats with confirmed, accurate NTS stimulation sites are presented here.

\section{Statistical Analysis}

The effects of hypotension on c-fos expression in NTS neurons were evaluated by counts of labeled neurons in the control and hypotension conditions, and comparison by a $t$-test. We analyzed the effects of NTS stimulation on ChBF, ChBVol, ChBVel and $\mathrm{ABP}$. For the time blocks prior to stimulation, we calculated the mean for ChBF, ChBVol, ChBVel and ABP, which served as the baseline level for these parameters against which to evaluate stimulation effects. For an analysis of the response to NTS activation, we compared the response during the $7 \mathrm{~s}$ of NTS stimulation to the response for that parameter in the $7 \mathrm{~s}$ prior to stimulation, using $t$-tests. For the time blocks during NTS activation in the mapping studies, we determined mean response for each rat, and then calculated group means. For the NPA studies, peak response, latency of the response (defined as $50 \%$ above basal) and time to peak during NTS activation were also determined. Data are presented separately for the NTS responses before NPA administration (pre-NPA) and after NPA administration (NPA), and the raw data analyzed by 2-way ANOVA with subsequent planned comparisons between groups by Fisher PLSD tests. Regression analysis was used to evaluate the contributions of ABP, ChBVol and ChBVel changes to $\mathrm{ChBF}$ changes. Group results are presented as mean \pm SEM.

\section{RESULTS}

\section{Baroresponsive Subdivisions of NTS}

Sustained ABP 25-30\% below basal was produced by $4-7 \mathrm{ml}$ blood withdrawal to identify hypotensive responsive neurons in NTS, as assessed by c-fos immunolabeling. Counts of labeled neurons showed that the abundance of $\mathrm{c}$-fos+ neurons following hypotension was tripled in baroreceptive NTS, notably in the dorsal, solitary, intermediate and ventral subdivisions of more caudal NTS (Figure 1). The increase was statistically significant $(p<0.043)$. The distribution of $c$-fos+ neurons in NTS after hypotension that we observed matches that described by others (Rogers et al., 1993; Mayne et al., 1998), and overlaps the terminal field within NTS of aortic and carotid sinus baroreceptor afferent input (Figure 2) via the vagus and glossopharyngeal nerves (Ciriello, 1983; Housley et al., 1987; Altschuler et al., 1989). Moreover, the distribution
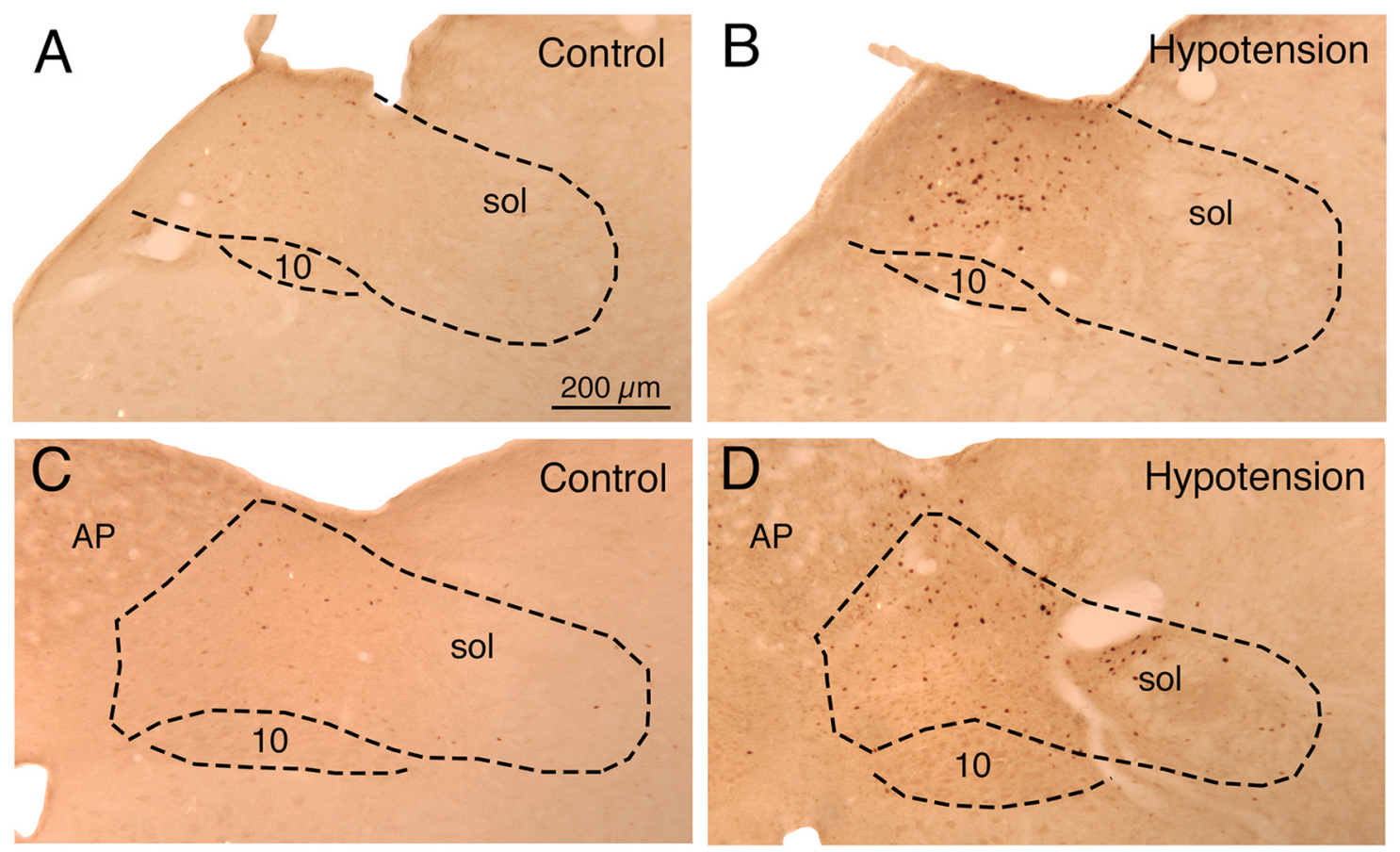

FIGURE 1 | Images showing the distribution of c-fos immunolabeling in: (1) nucleus of the solitary tract (NTS) just anterior to the area postrema (AP) in a sham hypotension rat (A) and a hypotensive rat (B); and (2) NTS at the level of AP in a sham hypotension rat (C) and a hypotensive rat (D). The NTS is outlined, as is the vagal motor nucleus (10), and the location of the solitary tract (sol) is indicated. Note that hypotension induces c-fos in any neurons around and medial to the solitary tract. All images are at the same magnification. 


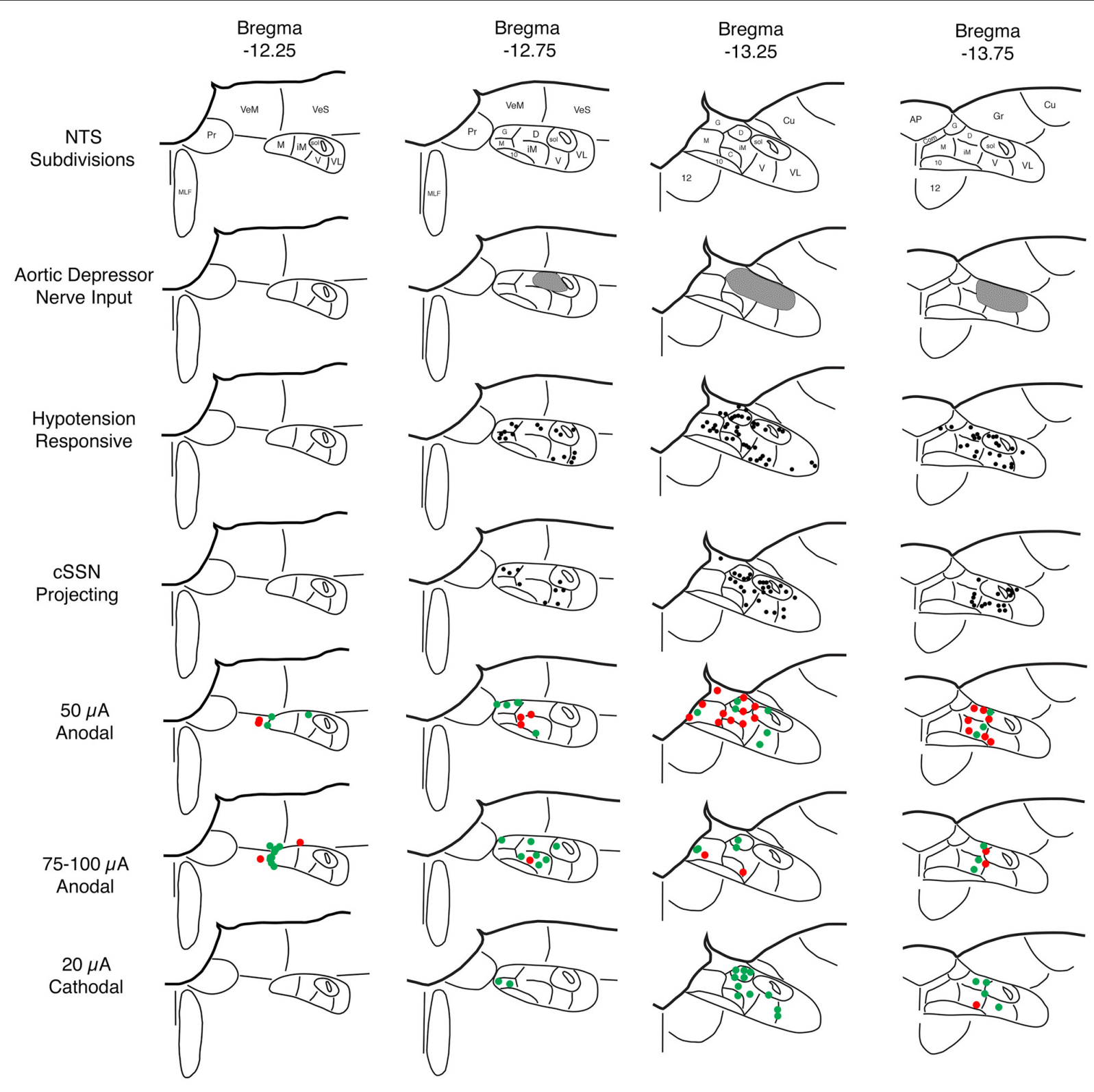

FIGURE 2 | Schematic illustration showing: (1) the NTS subdivisions (row 1); (2) the distribution of the aortic depressor nerve (ADN) input to superior salivatory nucleus (SSN) according to published studies by others (row 2; Ciriello, 1983; Housley et al., 1987; Altschuler et al., 1989); (3) the distribution of c-fos immunolabeling in NTS induced by hypotension as indicated in the present study and as consistent with prior reports by others (row 3; Rogers et al., 1993; Mayne et al., 1998); (4) the distribution of neurons in NTS that project to choroidal SSN (cSSN) as revealed from our prior studies involving intrachoroidal injection of pseudorabies virus (row 4; Li et al., 2015); (5) the distribution of sites within NTS yielding a significant choroidal blood flow (ChBF) increase (green) or no significant ChBF increase (red) in response to $50 \mu \mathrm{A}$ anodal current pulses (row 5); (6) the distribution of sites within NTS yielding a significant ChBF increase (green) or no significant ChBF increase (red) in response to 70-100 $\mu$ A anodal current pulses (row 6); and (7) the distribution of sites within NTS yielding a significant ChBF increase (green) or no significant ChBF increase (red) in response to $20 \mu \mathrm{A}$ cathodal current pulses (row 7). Note that hypotension responsive and cSSN neurons overlap one another, and both overlap but extend beyond the zone of ADN input. Thus, hypotension responsive and cSSN neurons either extend their dendrites into the ADN-receptive zone and/or receive input from AND-receptive neurons to account for the baroresponsiveness. The stimulation data indicate that ChBF increases can be driven by anodal stimulation in rostromedial NTS along the course of the axons traveling from NTS to cSSN and by cathodal stimulation within the region enriched in hypotension responsive and cSSN projecting neurons.

of c-fos+ neurons in NTS after hypotension matches the distribution of neurons within NTS that project to prechoroidal SSN (Figure 2) identified in our own work (Li et al., 2015).
These findings are consistent with the notion that the NTS input to prechoroidal SSN is responsive to systemic ABP signals. Note that baroresponsive/SSN-projecting NTS neurons 
extend outside of the NTS zone receiving baroreceptive input, implying that neurons in baroreceptive NTS may project outside of this territory to neurons in baroresponsive/SSNprojecting NTS.

\section{Identification of NTS Sites Yielding ChBF Increases -50 $\mu$ A Anodal Stimulation}

Electrical activation of NTS with $50 \mu \mathrm{A}$ anodal current pulses revealed loci that yielded $\mathrm{ChBF}$ increases by at least $10 \%$ above baseline (defined as effective sites), and others that did not (termed ineffective sites; Figures 2, 3). No differences were present in basal choroidal parameters or ABP for these sites (Table 1). The mean $\mathrm{ChBF}$ increases for the 17 effective sites with $50 \mu \mathrm{A}$ anodal pulses were significantly elevated above baseline by $43.9 \% \pm 12.7 \%(p=0.0021)$, while there was no significant mean ChBF increase $(1.0 \% \pm 1.1 \%)$ for the 23 ineffective sites $(p=0.3502$; Figures $2-4)$. Both effective and ineffective sites were observed in the baroreceptive and baroresponsive NTS subdivisions with $50 \mu \mathrm{A}$ stimulation (Figure 2). Effective sites were most consistently seen in dorsomedial and rostral NTS, which coincides with the path of axons coursing from baroreceptive/baroresponsive NTS to prechoroidal SSN (Figure 5). The increases for the effective sites were accompanied by significant $15.7 \% \pm 3.4 \%$ increases in ChBVol $\left(p=0.01 \times 10^{3}\right)$ and $21.3 \% \pm 10.2 \%$ increases in ChBVel $(p=0.0462)$. As addressed in the "Discussion Section", we interpret the volume increases for the effective sites to reflect vasodilation of choroidal vessels, and the velocity increases may largely reflect vasodilation of intraorbital choroidal feeder vessels. By contrast, no significant changes were seen for $\mathrm{ChBVol}$ $(+1.0 \% \pm 1.0 \%, p=0.3509)$ or ChBVel $(-0.3 \% \pm 1.1 \%$, $p=0.8100)$ with the ineffective $50 \mu \mathrm{A}$ sites. Mean systemic $\mathrm{ABP}$ with effective $50 \mu \mathrm{A}$ stimulation sites, by contrast to the choroidal effects, was only $0.5 \% \pm 0.3 \%$ above basal, which was not significant ( $p=0.0940$; Figures 3, 4). Similarly, mean systemic ABP with ineffective $50 \mu \mathrm{A}$ NTS sites was $1.3 \% \pm 0.8 \%$ above basal (Figure 4), which was not significant ( $p=0.1035$ ). Thus, as ABP alterations caused by NTS activation were not significant, increased perfusion pressure due to increased $A B P$ is unlikely to be a driver of the ChBF increases with $50 \mu \mathrm{A}$ stimulation.
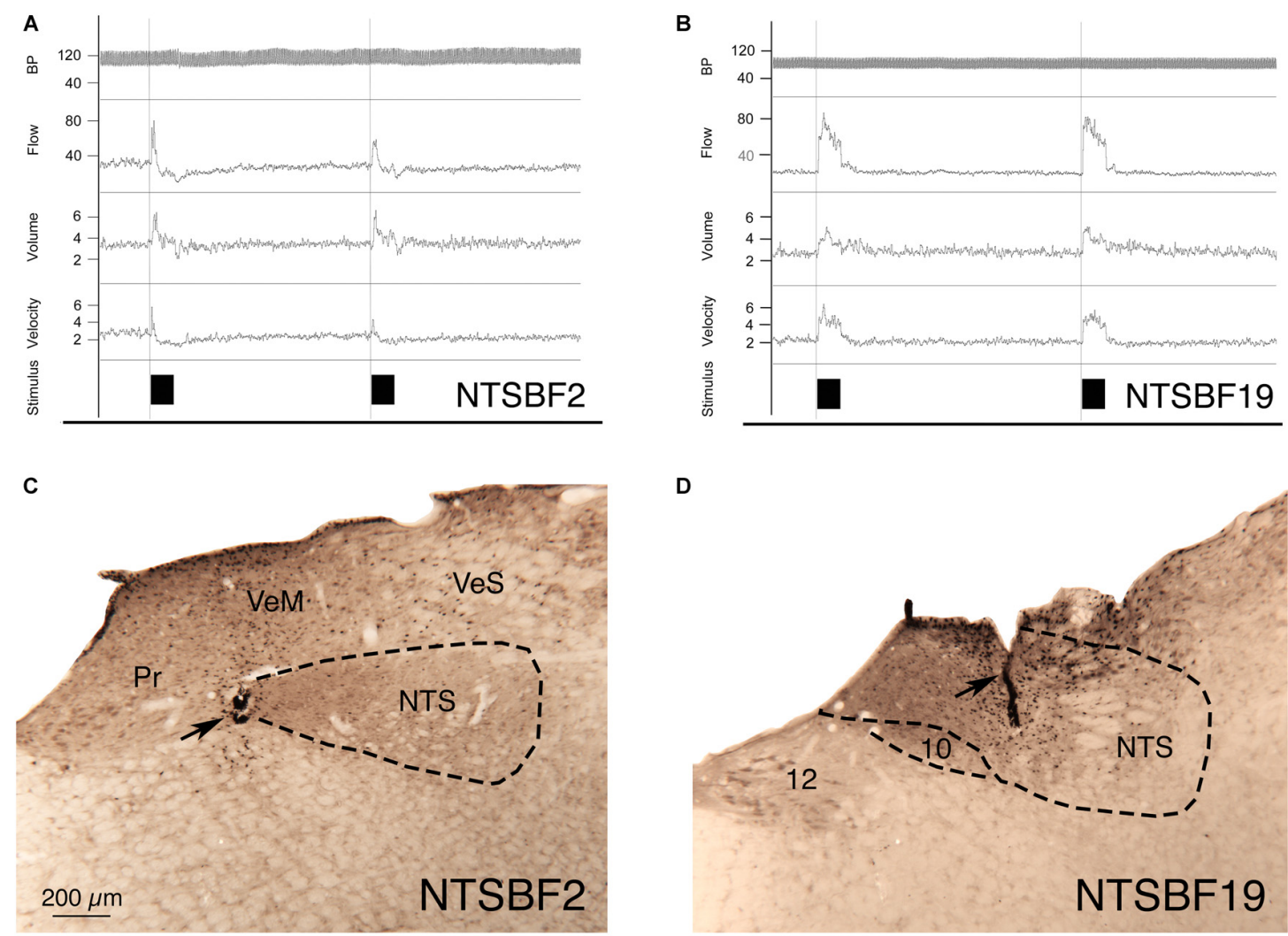

D

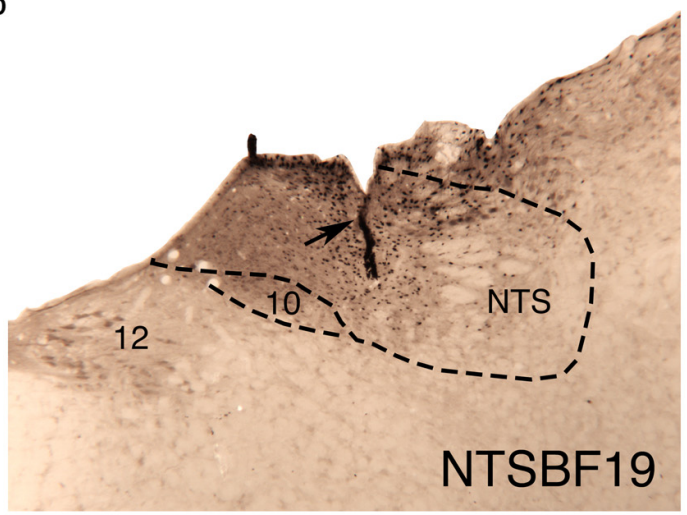

FIGURE 3 | Choroidal vasodilation with NTS stimulation from an illustrative case (NTSBF2) with $100 \mu$ A anodal stimulation (A,C), and an illustrative case (NTSBF19) with $20 \mu$ A cathodal stimulation (B,D). (A) Shows recording traces for arterial blood pressure (ABP), ChBF, volume and velocity in response to $7 \mathrm{~s}$ anodal current pulse trains for NTSBF2 at the rostromedial NTS site shown by the arrow in (C). During each NTS stimulation, ChBF, ChBVol, and choroidal blood velocity (ChBVel) increased for NTSBF2, but ABP did not. The effective stimulation site shown in this case is at the medial edge of the fiber tract traveling within NTS to cSSN. (B) Shows recording traces for ABP, ChBF, volume and velocity in response to $7 \mathrm{~s}$ cathodal current pulse trains for NTSBF19 at the more caudocentral NTS site shown by the arrow in (D). During each NTS stimulation, ChBF, ChBVol, and ChBVel increased for NTSBF19, but ABP did not. The effective stimulation site shown in this case is within a part of NTS enriched in neurons projecting to cSSN. The magnification is the same in $(\mathbf{A}, \mathbf{B})$. 
TABLE 1 | Basal choroidal parameters (ChBF, ChBVol and ChBVel) and basal arterial blood pressure (ABP) for the different sets of nucleus of the solitary tract (NTS) stimulation sites examined.

\begin{tabular}{|c|c|c|c|c|c|}
\hline Stimulation group & \# of sites & Basal ChBF & Basal ChBVol & Basal ChBVel & Basal ABP \\
\hline Effective $50 \mu \mathrm{A}$ anodal sites & 17 & $18.0 \pm 1.21$ & $2.98 \pm 0.17$ & $1.82 \pm 0.07$ & $104.7 \pm 4.20$ \\
\hline Ineffective $50 \mu \mathrm{A}$ anodal sites & 23 & $19.0 \pm 1.17$ & $2.94 \pm 0.12$ & $1.91 \pm 0.08$ & $101.9 \pm 1.78$ \\
\hline Effective $70-100 \mu \mathrm{A}$ anodal sites & 24 & $19.4 \pm 1.32$ & $3.30 \pm 0.09$ & $1.80 \pm 0.09$ & $102.2 \pm 1.86$ \\
\hline Ineffective $70-100 \mu \mathrm{A}$ anodal sites & 7 & $19.4 \pm 2.20$ & $3.40 \pm 0.24$ & $1.68 \pm 0.10$ & $95.7 \pm 1.86$ \\
\hline Effective $-20 \mu \mathrm{A}$ cathodal sites & 18 & $15.0 \pm 0.89$ & $2.84 \pm 0.08$ & $1.60 \pm 0.07$ & $91.4 \pm 5.31$ \\
\hline Ineffective $-20 \mu \mathrm{A}$ cathodal sites & 1 & 18.7 & 3.23 & 1.75 & 128.6 \\
\hline pre-NPA effective $100 \mu \mathrm{A}$ anodal sites & 8 & $21.1 \pm 2.58$ & $3.09 \pm 0.15$ & $2.06 \pm 0.21$ & $97.9 \pm 5.76$ \\
\hline With-NPA effective $100 \mu \mathrm{A}$ anodal sites & 8 & $16.8 \pm 1.70$ & $3.15 \pm 0.11$ & $1.81 \pm 0.19$ & $95.3 \pm 5.78$ \\
\hline
\end{tabular}

\section{Identification of NTS Sites Yielding ChBF Increases -70 to $100 \mu \mathrm{A}$ Anodal Stimulation}

Similar results were seen with 70-100 $\mu$ A current pulses, except that the mean increase above baseline for the 24 effective NTS sites was greater than for $50 \mu \mathrm{A}$ pulses $-73.5 \% \pm 9.5 \%$ for $70-100 \mu \mathrm{A}\left(p=0.02 \times 10^{6}\right)$ vs. $43.9 \% \pm 12.7 \%$ for $50 \mu \mathrm{A}$ (Figures 2, 4). The effective sites were most commonly seen in the dorsal and intermediate subdivisions of NTS, and along the course of the NST axons traveling to prechoroidal SSN (Figure 2). Relatively fewer ineffective sites were observed with $70-100 \mu \mathrm{A}$ anodal current pulses than with $50 \mu \mathrm{A}$ pulses. No differences were present in basal choroidal parameters or $\mathrm{ABP}$ for these sites (Table 1). For ineffective sites (Figure 4),
ChBF was not significantly changed from basal $(+3.0 \% \pm 1.8 \%$, $p=0.2348$ ). Neither stimulation of the effective NTS sites nor the ineffective sites yielded significant changes in mean $A B P$ (Figure 4)-namely, $+0.1 \% \pm 0.2 \%$ for effective $(p=0.9045)$ and $-0.1 \% \pm 1.3 \%$ for ineffective $(p=0.8814)$. Thus, the ChBF increases with effective 70-100 $\mu$ A NTS sites were not generated by effects of stimulation on ABP. Given that the ChBF increases for the 70-100 $\mu \mathrm{A}$ effective sites were accompanied by significant mean $21.5 \% \pm 2.9 \%$ increases in $\mathrm{ChBVol}\left(p=0.01 \times 10^{6}\right)$ and mean $38.8 \% \pm 6.2 \%$ increases in ChBVel $\left(p=0.011 \times 10^{3}\right)$, the $\mathrm{ChBF}$ increases appear to be driven by vasodilation of choroidal vessels and in all likelihood intraorbital choroidal feeder vessels. The absence of a significant ChBF increase for ineffective $70-100 \mu \mathrm{A}$ sites is consistent with the absence of

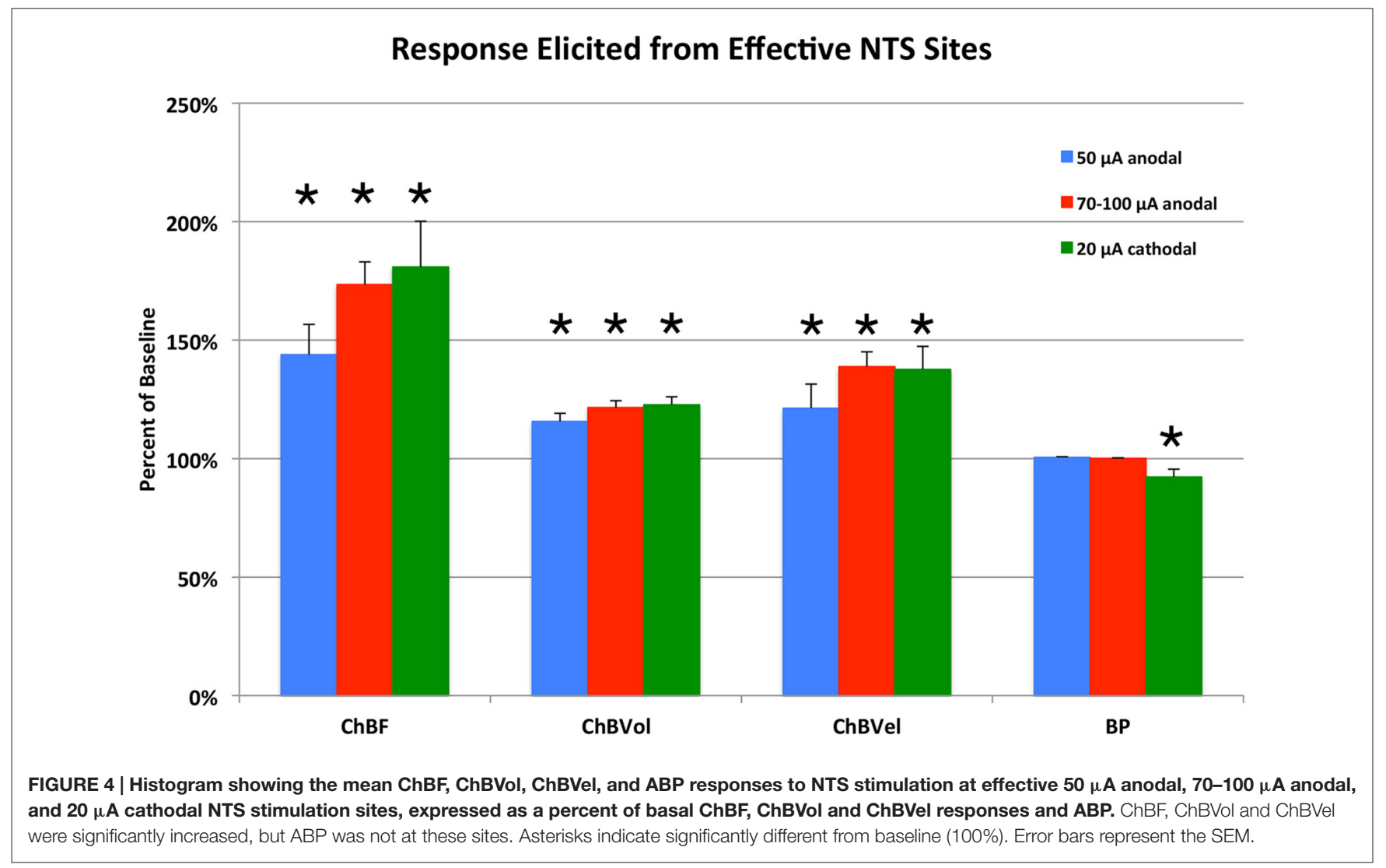



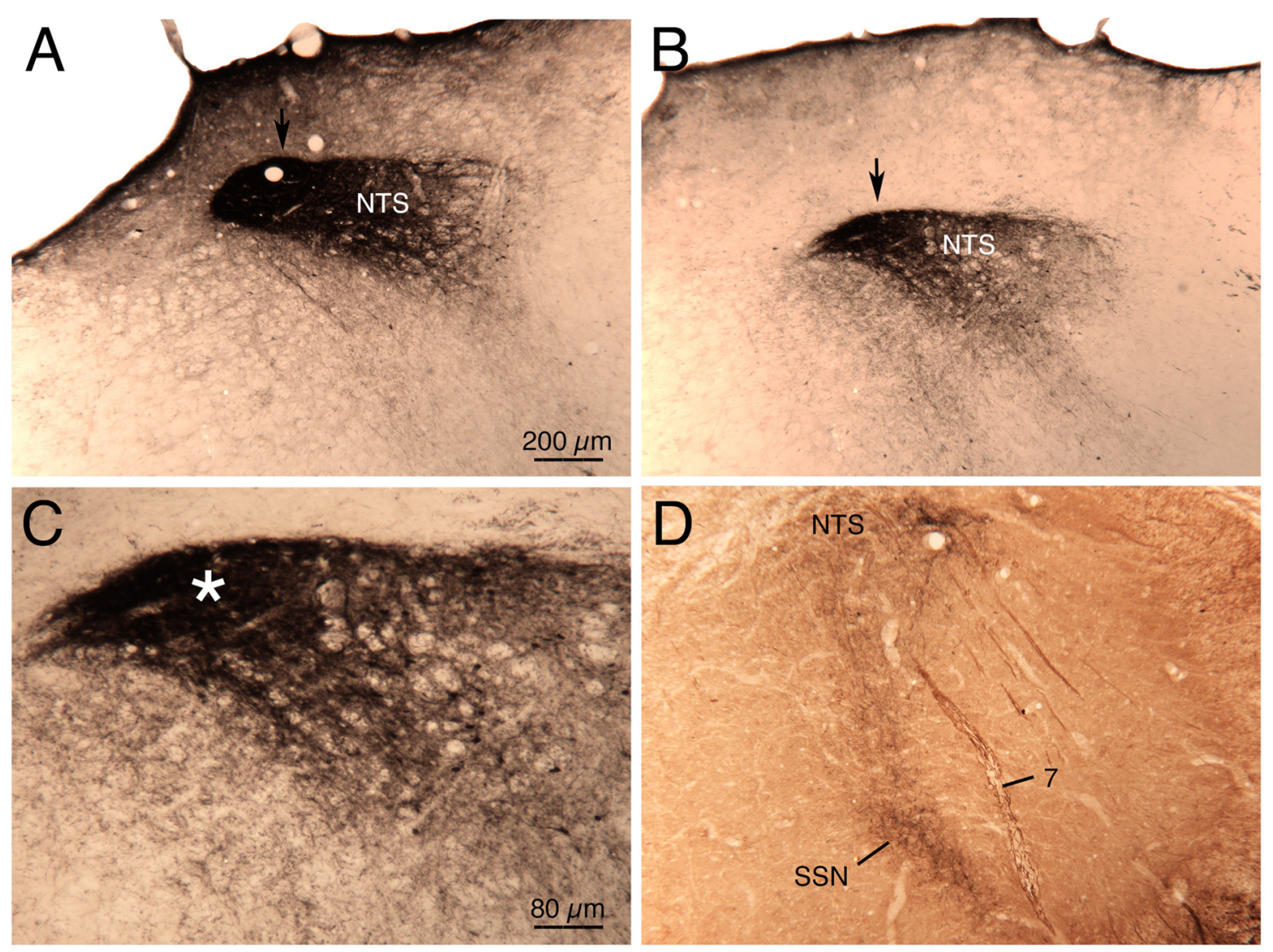

FIGURE 5 | Images showing the course of the axons from baroreceptive NTS coursing to prechoroidal SSN, as visualized in a previously described case (Li et al., 2010, 2015) in which biotinylated dextran amine was injected into NTS just anterior to the AP. Images (A,B,D) show increasingly rostral levels through NTS, and the NTS axons are indicated by an arrow in (A,B). Higher magnification view of the axons shown in image (B) is shown in image (C) asterisk). In image (D), two-color DAB labeling has been performed to simultaneously detect the neurons (brown) of choroidal SSN for their enrichment in neuronal nitric oxide (NO) synthase, and the axons (black) coursing from NTS to the SSN. The magnification is the same in (A,B,D).

significant changes in ChBVol $(+1.8 \% \pm 1.5 \%, p=0.3115)$ or ChBVel $(+2.5 \% \pm 1.6 \%, p=0.8814)$ for these sites.

\section{Identification of NTS Sites Yielding ChBF Increases-20 $\mu$ A Cathodal Stimulation}

Because anodal stimulation tended to drive $\mathrm{ChBF}$ increases most effectively from the axons traveling from NTS to prechoroidal SSN, we used cathodal stimulation of NTS in a second set of rats to provide better localization of the perikarya that drive $\mathrm{ChBF}$ increases from within NTS. We focused our penetrations on the subdivisions of NTS that receive baroreceptive input and are baroresponsive. Unlike with anodal stimulation, the 18 effective $20 \mu \mathrm{A}$ cathodal NTS sites were localized primarily to baroreceptive/baroresponsive NTS-namely the dorsal, intermediate, ventral and solitary subdivisions (Figure 2). Only one ineffective site was found in this region with $20 \mu \mathrm{A}$ cathodal stimulation (Figure 2). Basal choroidal parameters were similar for the effective sites to those for the one ineffective site, although ABP was much higher for the ineffective site (Table 1). A significant mean
$80.9 \% \pm 19.3 \%$ ChBF increase was observed for the effective sites ( $p=0.00054)$, while for the ineffective site ChBF was only $+2.6 \%$ above baseline (Figure 4). Similarly, ChBVol was $+22.7 \% \pm 3.4 \%$ above baseline $\left(p=0.03 \times 10^{4}\right)$ and ChBVel was $+37.6 \% \pm 9.7 \%$ above baseline $(p=0.0018)$. The ineffective site showed a large ChBVol decrease $(-8.4 \%)$ but an equally large ChBVel increase $(+10.7 \%)$, suggesting that this site had a mixed effect on ChBF. Although there was no ABP change with the ineffective site $(+0.6 \%)$, a significant $7.7 \% \pm 3.2 \%$ decrease in ABP was seen with $20 \mu \mathrm{A}$ cathodal stimulation for effective NTS sites $(p=0.0295$; Figure 4).

\section{Control Stimulation}

To further evaluate the specificity of ChBF increases with NTS stimulation, we examined the effects of stimulation of cell groups around NTS. Activation of the hypoglossal nucleus ventromedial to NTS, the vagal motor nucleus along the lower medial border of NTS, or the cuneate or the medial and superior vestibular nuclei above NTS with anodal or cathodal stimulation commonly yielded eye, jaw or tongue movement, as well as variable effects 
on choroidal parameters and ABP. By contrast, such movements were rarely observed during NTS stimulation, and in those rare instances in which they were, the data were excluded, since the movements could produce artificial ChBF results.

\section{NTS Stimulation - Choroidal Blood Flow Dynamics}

We used regression analysis to further assess the contributions of ABP, ChBVol and ChBVel to the observed ChBF increases with activation of effective NTS sites. For the 41 effective NTS sites observed with 50-100 $\mu \mathrm{A}$ anodal pulses, the ABP change $(+0.32 \%)$ remained nonsignificant, and showed no significant positive correlation with $\mathrm{ChBF}$, ChBVol, or ChBVel either prior to or during NTS stimulation. ChBF during 50-100 $\mu \mathrm{A}$ anodal NTS stimulation was much more highly correlated with ChBVel $(r=0.8998)$ than with ChBVol $(r=0.3198)$, although both correlations were significant. For the 18 effective $20 \mu \mathrm{A}$ cathodal NTS sites, ChBVel was again significantly and highly correlated $(r=0.9845)$ with ChBF. In this case, however, ChBVol was also significantly and highly correlated with $\mathrm{ChBF}(r=0.7279)$. Thus, intrachoroidal vasodilation was a greater contributor to ChBF increases with cathodal than anodal stimulation. Although a significant decline in ABP was observed during $20 \mu \mathrm{A}$ cathodal NTS stimulation that yielded $\mathrm{ChBF}$ increases, the change in $\mathrm{ABP}$ was not significantly correlated with $\mathrm{ChBF}$, ChBVol, or ChBVel. Thus, for cathodal stimulation as well as for anodal stimulation, any effects of NTS activation on ABP did not notably influence ChBF.

\section{NTS Stimulation - Pre-NPA Choroidal Blood Flow Effects}

In eight separate rats, we examined the effects of nNOS inhibition on NTS-elicited ChBF increases. For these studies, $100 \mu \mathrm{A}$ anodal current pulses were delivered to NTS and yielded robust $\mathrm{ChBF}$ increases. The relative $\mathrm{ChBF}, \mathrm{ChBV}$ ol, ChBVel and ABP changes prior to, during and after stimulation were plotted for these rats to further examine the hemodynamics of the effect of electrical stimulation of NTS on ChBF, and as a baseline against which to compare NPA effects. NTS stimulation yielded significant mean increases above basal ChBF $(+133.9 \% \pm 30.3 \%$, $p=0.002)$, basal ChBVol $(+48.6 \% \pm 18.8 \%, p=0.0353)$, and basal ChBVel $(+81.1 \% \pm 27.1 \%, p=0.0149)$, but no significant change in mean ABP from baseline $(+2.6 \%, p=0.7757)$ during the 7-s stimulation period (Figures 6,7). The ChBF increase reached $50 \%$ above baseline by $0.94 \mathrm{~s}$ after current onset, and peaked at $2.99 \mathrm{~s}$ after stimulus onset. The ChBVol increased slightly more rapidly initially than did ChBVel, but their peaks were similar and occurred around $3.0 \mathrm{~s}$ after current onset. The NTS-stimulation-evoked increases in ChBF, ChBVol and ChBVel returned to baseline following stimulation offset, with a slight transient undershoot for $\mathrm{ChBF}$ and $\mathrm{ChBVel}$ but not ChBVol. NTS activation for these cases yielded a slight, gradual rise in $\mathrm{ABP}$, resulting in a peak $\mathrm{ABP}(+15.9 \% \pm 3.8 \%)$ at $4.45 \mathrm{~s}$ after onset of NTS stimulation onset that was, nonetheless, not significantly greater $(p=0.2596)$ than basal ABP. The
ABP quickly returned to basal levels upon stimulation offset (Figure 7).

\section{Effect of nNOS Inhibition on ChBF Increase during NTS Stimulation}

Consistent with its action on nNOS but not endothelial nitric oxide synthase (eNOS), NPA had no significant effect on basal $\mathrm{ABP}(-2.5 \% \pm 3.1 \%, p=0.7549$; Table 1; Figure 6). NPA also did not significantly alter mean basal ChBF $(-16.3 \% \pm 8.6 \%$, $p=0.4836$ ), although mean $\mathrm{ChBF}$ after NPA tended to be consistently lower over time (Table 1; Figures 6, 8). Basal ChBVol $(+2.2 \% \pm 2.4 \%, p=0.9392)$ and ChBVel $(-11.3 \% \pm 6.9 \%, p=0.6790)$ were also not significantly affected by NPA (Table 1). The effect of stimulation of NTS on mean and peak $\mathrm{ChBF}, \mathrm{ChBVol}$ and/or ChBVel was, however, substantially reduced after NPA. For example, the mean ChBF increase with NPA was $48.9 \% \pm 18.0 \%$ of that prior to NPA. Moreover, the mean ChBF during NTS stimulation after NPA treatment was no longer significantly above baseline ( $p=0.2303$ ). Additionally, the mean ChBF during NTS stimulation was significantly less after NPA than prior to NPA $(p=0.0008)$. Similarly, the peak ChBF with NPA was only $50.7 \% \pm 19.0 \%$ of that during pre-NPA. Although the peak ChBF during NPA remained significantly above NPA baseline $(p=0.0245)$, the pre-NPA vs. NPA difference in peak $\mathrm{ChBF}$ increase with NTS stimulation was highly significant $\left(p=0.028 \times 10^{3}\right.$; Figures 6, 8). The ChBVol increase seemed comparably attenuated by NPA as the ChBVel increase during NTS stimulation (Figures 6, 9). For example, the mean ChBVol increase was only $34.5 \% \pm 10.0 \%$ of pre-NPA, and the ChBVol after NPA was no longer significantly greater than basal ChBVol ( $p=0.7227)$. Similarly, the mean ChBVel increase evoked by NTS activation after NPA administration was $28.8 \% \pm 10.5 \%$ of pre-NPA, and was also no longer significantly greater than basal ChBVel after NPA $(p=0.7207)$. The response to NTS activation was also significantly less than observed prior to NPA $(p=0.0168)$. Thus, the effects of NPA on ChBF were driven by its inhibitory effects on both ChBVel and ChBVol. In general, NPA appeared to slightly slow the onset of choroidal responses and the time to peak (Figure 7).

\section{DISCUSSION}

This study shows that ChBF increases significantly upon activation of the baroresponsive part of NTS or its output fibers to prechoroidal SSN, and the increase in ChBF elicited by NTS activation is attenuated by the neuronal NOS inhibitor NPA. The ChBF increase appears to be mediated by vasodilation of orbital and choroidal blood vessels, but not by any effects of NTS activation on systemic BP. Our results indicate that NTS neurons projecting to choroidal SSN do mediate increases in $\mathrm{ChBF}$. As these neurons tend to reside in the baroresponsive part of NTS, our results suggest a role of baroreceptive NTS in the well demonstrated BP-dependent regulation of ChBF (Kiel and Shepherd, 1992; Lovasik et al., 2003; Reiner et al., 2003, 2010b, 
A

Responses Elicited with NTS Stimulation - pre NPA

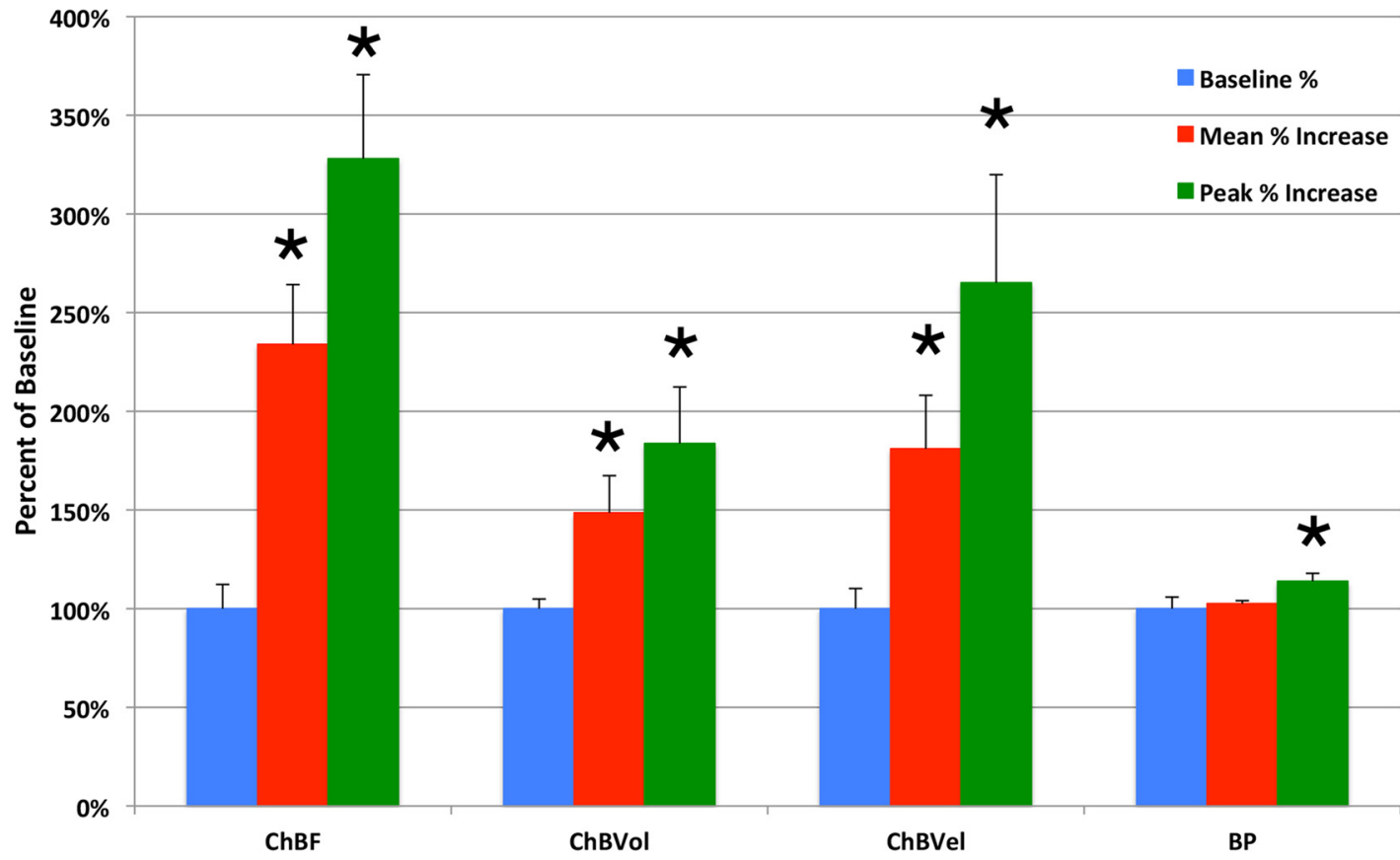

B

Responses Elicited with NTS Stimulation - during NPA

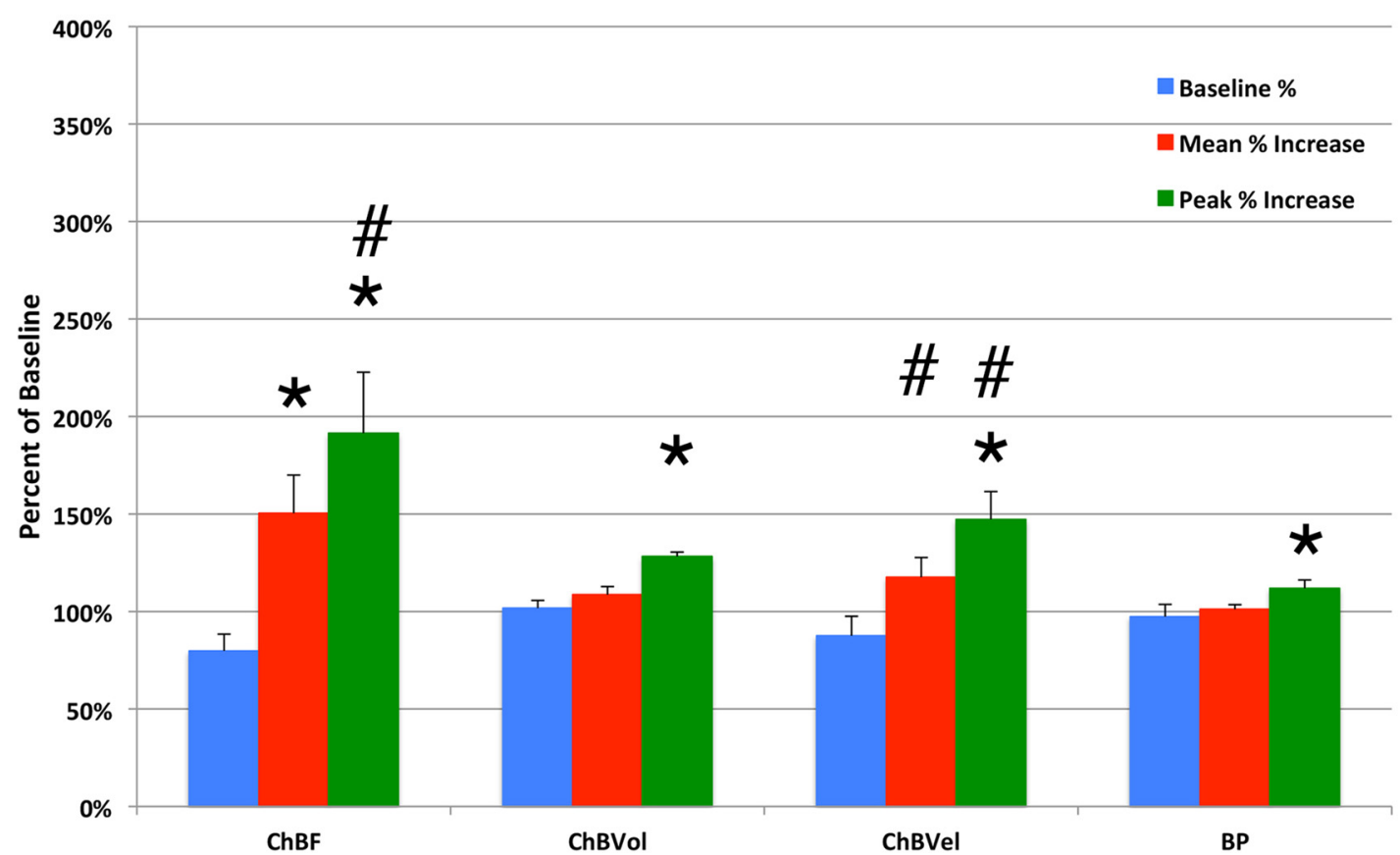

FIGURE 6 | Histogram showing the mean and peak ChBF, ChBVol and ChBVel responses and ABP responses to NTS stimulation at effective $100 \mu \mathrm{A}$ anodal stimulation sites, expressed as a percent of basal ChBF, ChBVol and ChBVel responses and ABP, prior to $\mathbf{N}^{\omega}$-propyl-I-arginine (NPA)

administration (A) and after NPA administration (B). Note that NTS stimulation prior to NPA yielded significant ChBF, ChBVol and ChBVel increases but had no effect on ABP, and that NPA attenuated the ChBF, ChBVol and ChBVel increases but had no effect on ABP. Asterisks indicate significantly different from baseline, and ampersands indicate significantly less than pre-NPA. Error bars represent the SEM. 


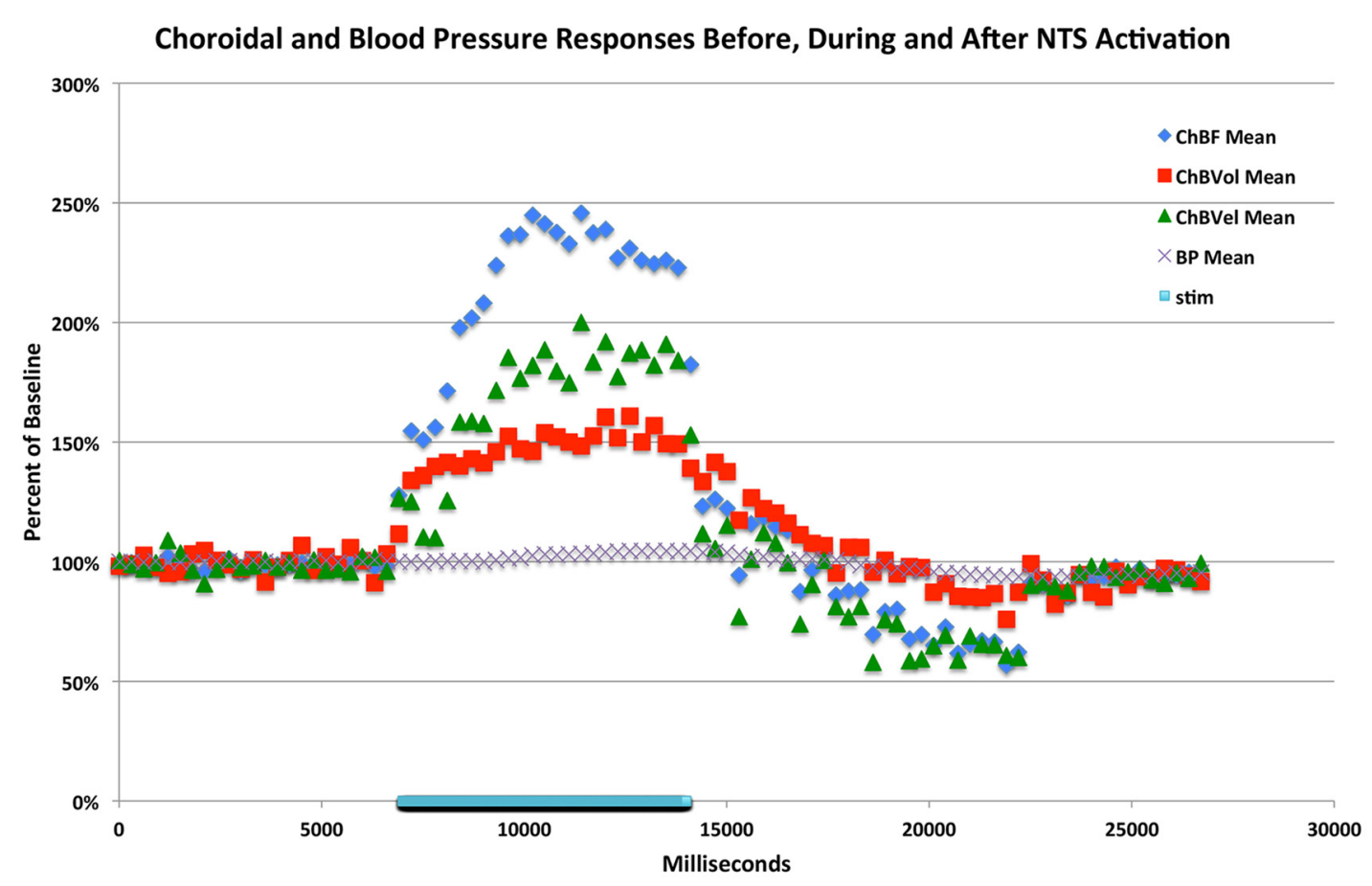

FIGURE 7 | Graph showing the time course of the mean ChBF, ChBVol, ChBVel and ABP responses to stimulation at effective anodal NTS sites $(\boldsymbol{n}=\boldsymbol{x})$. The blue bar marks the stimulation period. Each data point is the mean for a $333 \mathrm{~ms}$ interval, and ChBF, ChBVol, ChBVel and ABP responses are all expressed as percent of basal. The rapid ChBF increases are driven by rapid increases in both ChBVel and volume.

2011), which we refer to as baroregulation. These considerations are discussed in more detail below.

\section{NTS and ChBF Baroregulation Circuitry}

The parasympathetic PPG innervates orbital vessels feeding the choroid (including the posterior ciliary arteries directly entering the choroid as well as the ophthalmic artery from which they arise), as well as choroidal vessels themselves (Stone et al., 1987; Cuthbertson et al., 1997, 2003; Jablonski et al., 2007; Reiner et al., 2012). The preganglionic neurons regulating the PPG reside in the SSN, the autonomic component of the facial motor complex in the hindbrain (Cuthbertson et al., 2003). In prior studies on the inputs to the SSN in rats, we found that a major input arose from the NTS of the medulla (Li et al., 2010), and in more recent studies we have demonstrated that the NTS regions that project directly to the ChBF-control neurons of SSN overlap the parts of NTS receiving cardiovascular afferents conveying $\mathrm{ABP}$ information (Figure 2; Li et al., 2015). The present study directly examined the subdivision-specific influence of NTS on the ipsilateral ChBF using LDF, and showed that activation of specific parts of NTS causes increases in ChBF. These regions include the part of NTS receiving direct baroreceptive input (ipsilateral interstitial and dorsal NTS subnuclei), the parts of NTS surrounding baroreceptive NTS that respond to hypotension, and the course of the axons projecting from NTS to choroidal SSN. Anodal current was more effective in stimulating the axons projecting from NTS to choroidal SSN, while cathodal stimulation was more effective in revealing the location of the NTS neurons driving $\mathrm{ChBF}$ increases. This outcome is in keeping with the reported relative efficacies of anodal vs. cathodal stimulation in activating axons vs. perikarya, respectively (Ranck, 1975; Bagshaw and Evans, 1976; Merrill et al., 2005; Lu et al., 2008). Our finding that activation of baroresponsive NTS increases $\mathrm{ChBF}$ in the ipsilateral eye is consistent with our prior evidence that this projection is glutamatergic ( $\mathrm{Li}$ et al., 2015).

ChBVol was significantly increased during NTS stimulationdriven increases in $\mathrm{ChBF}$. This is consistent with the vasodilation of choroidal vessels by activation of the PPG input to them as a result of NTS stimulation, which would be expected to manifest as an increase in ChBVol, i.e., ChBVol as measured by LDF. ChBVel was also significantly increased during NTS activation that increased ChBF. Choroidal vasodilation (increased vessel cross-sectional area) should, however, in principle decrease ChBVel, since blood velocity is inversely related to vessel cross-sectional area. Thus, it is unclear how ChBVel could increase with choroidal vasodilation. One possibility is that vasodilation occurs as well in the arteries feeding the choroid (ophthalmic and ciliary), increasing the volume of blood flow into the choroid and thereby increasing ChBVel. This would be expected as the PPG also innervates these feeder arteries, and activation of these fibers as well as direct application of the vasodilators they release dilate them (Wiencke et al., 1994; Nilsson, 1996; Toda et al., 1998; Ayajiki et al., 2000; Jarajapu et al., 2004; Overend et al., 2005; Overend and Martin, 2007; 
A ChBF response to NTS stimulation before and during NPA

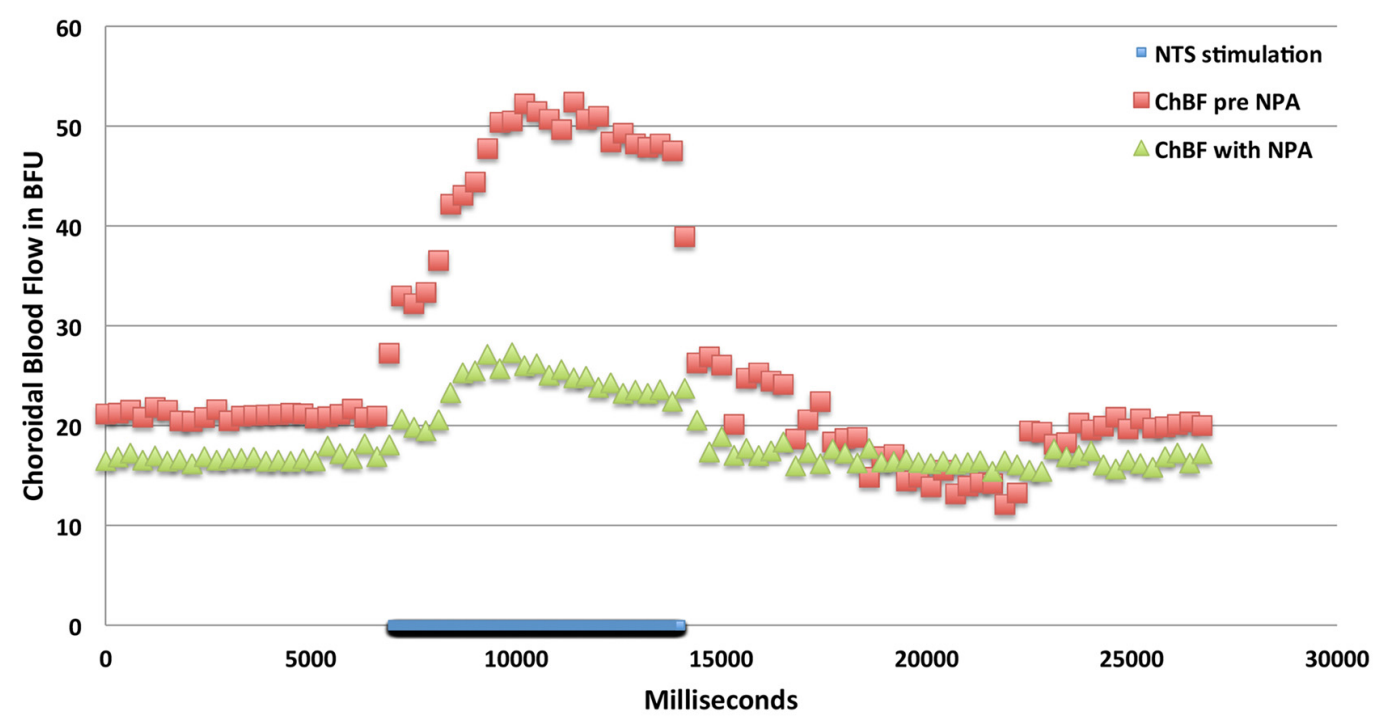

B Arterial BP response to NTS stimulation before and during NPA

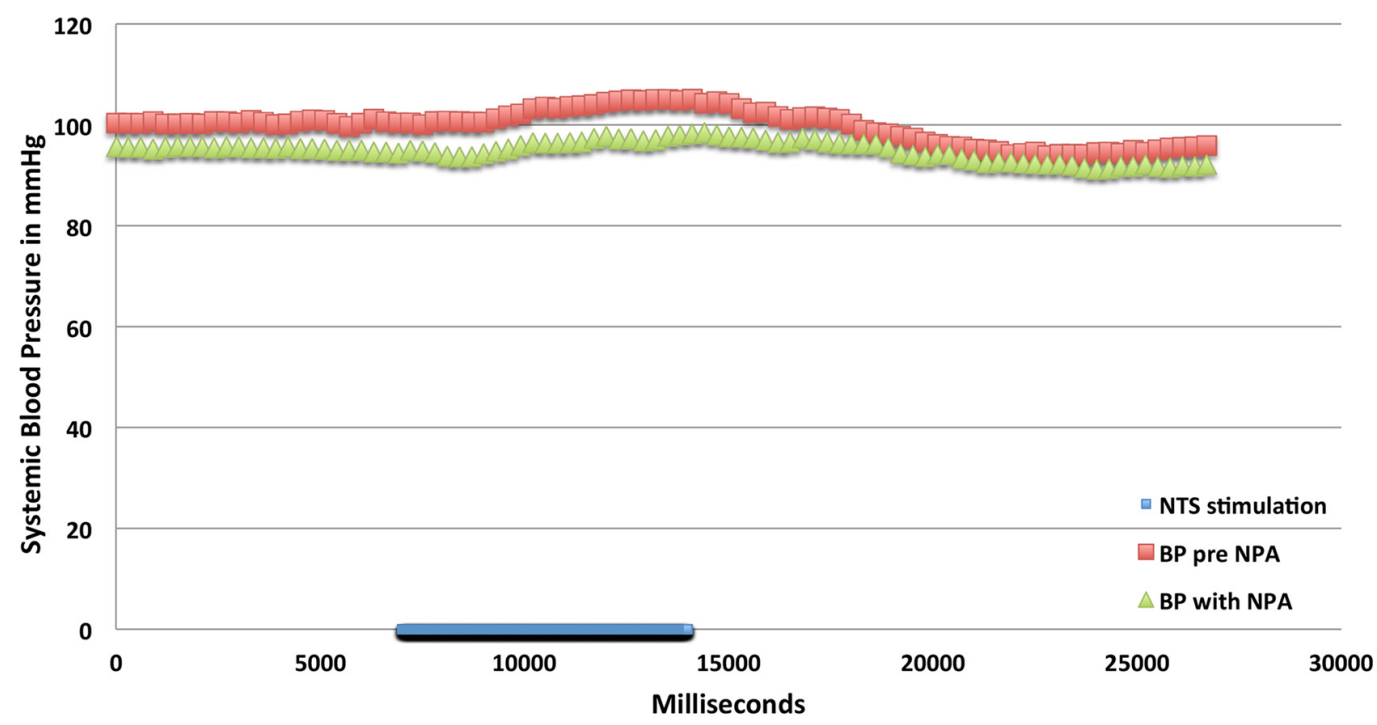

FIGURE 8 | Graphs showing the mean ChBF (A) and ABP (B) responses to stimulation at effective NTS sites prior to and after NPA administration $(\boldsymbol{n}=\boldsymbol{x})$. The blue bar marks the stimulation period. Each data point is the mean for $333 \mathrm{~ms}$. ChBF is expressed in relative blood flow units (BFU) and ABP in mm/Hg. Note that NPA significantly reduces the ChBF response to NTS activation, and has a consistent but insignificant depressive effect on ABP.

Reiner et al., 2012). Consistent with the impact of feeder vessel dilation on $\mathrm{ChBF}, \mathrm{ChBF}$ is correlated with flow in orbital vessels that directly (posterior ciliary arteries) or indirectly (ophthalmic artery) supply the choroid, according to a number of studies (Dorner et al., 2003; Zion et al., 2007; Rechtman et al., 2007; DeoKule et al., 2009; Pemp et al., 2012; Novais et al., 2015). Thus, the increases in $\mathrm{ChBVol}$ and $\mathrm{ChBVel}$ driving the increases in ChBF during NTS stimulation may have been driven by vasodilation in different vascular beds. The increase in ChBVol is likely to reflect choroidal vessel vasodilation, while the increase in ChBVel may have been caused by dilation of orbital feeder vessels. Alternatively, it is also possible that the increase in both $\mathrm{ChBV}$ ol and ChBVel that we observed entirely reflects complex dynamics of differential responses of choroidal arteries, veins and capillaries within the laser Doppler sampling volume to activation of the PPG input.

The PPG input to choroid and choroidal feeder vessels employs the vasodilators NO and VIP (Cuthbertson et al., 2003). 


\section{A ChBVol response to NTS stimulation before and during NPA}

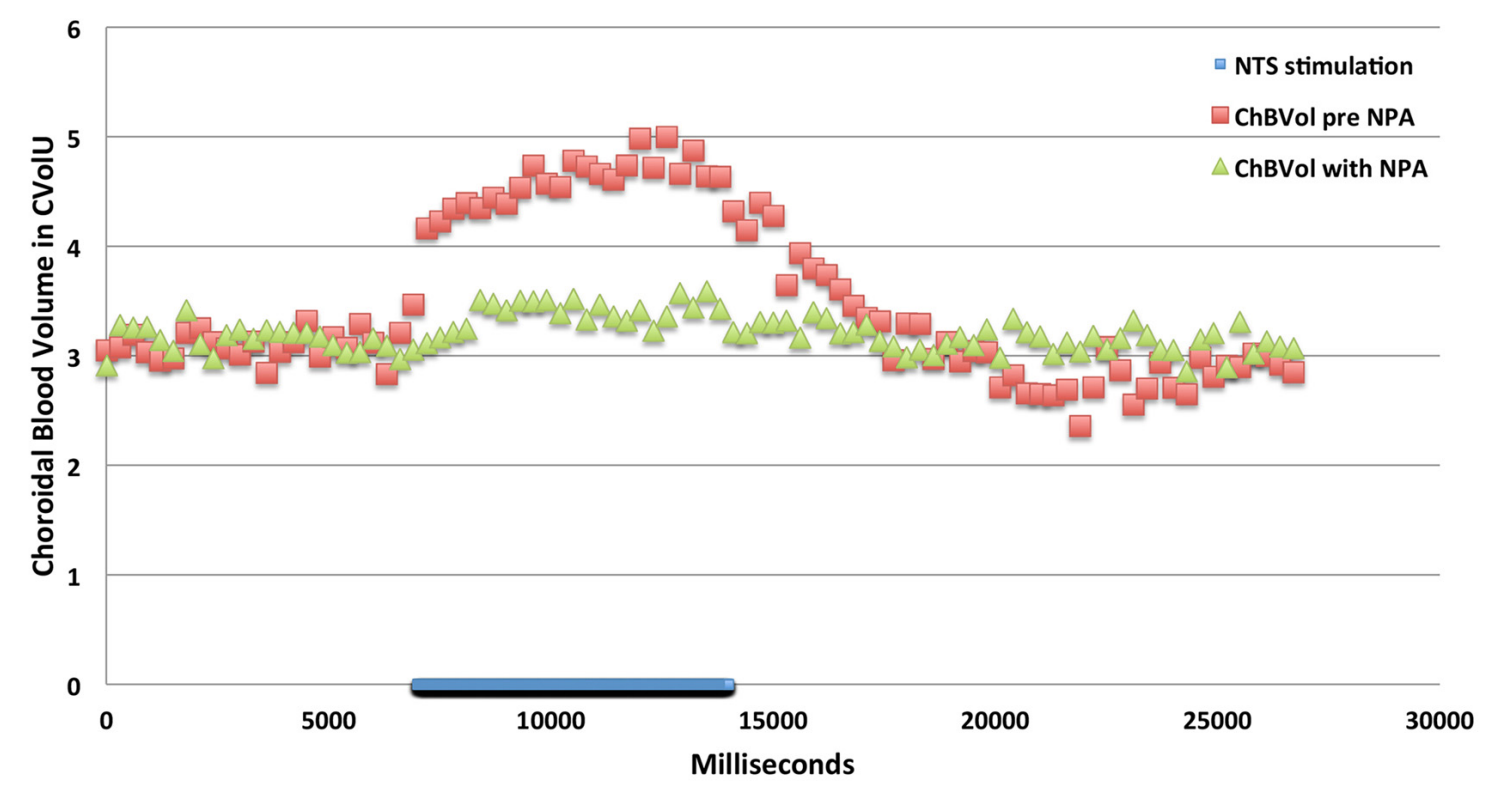

B ChBVel response to NTS stimulation before and during NPA

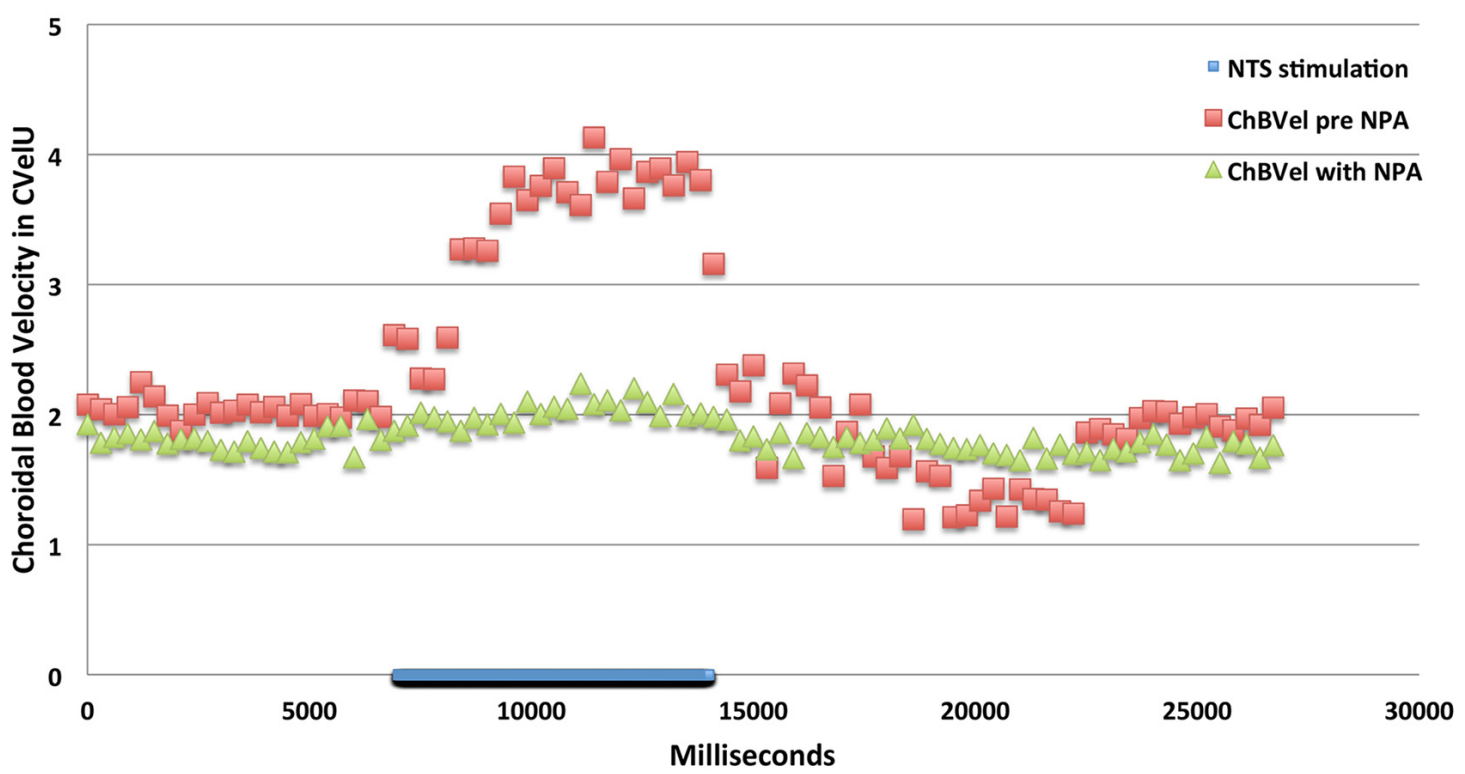

FIGURE 9 | Graphs showing the mean ChBVol (A) and ChBVel (B) responses to stimulation at effective NTS sites prior to and after NPA administration $(\boldsymbol{n}=\boldsymbol{x})$. The blue bar marks the stimulation period. Each data point is the mean for $333 \mathrm{~ms}$. ChBVol and ChBVel are expressed in relative units. Note that NPA significantly reduces both ChBVol and ChBVel responses to NTS activation.

As noted above, substantial evidence shows that both $\mathrm{NO}$ and VIP dilate orbital and choroidal vessels (Wiencke et al., 1994; Nilsson, 1996; Toda et al., 1998; Ayajiki et al., 2000; Jarajapu et al., 2004; Overend et al., 2005; Overend and Martin, 2007; Reiner et al., 2012). We found that NPA attenuated both the velocity-driven component and the volume-driven component of the ChBF increase. Given that NTS projects to the neurons of SSN that innervate PPG neurons innervating the entire geographic expanse of the choroid (Li et al., 2010) and that both NTS and SSN stimulation increase ChBF throughout choroid (Steinle et al., 2000; Fitzgerald et al. unpublished observations), our NPA data indicate that the ChBF increases elicited by NTS 
activation are likely to be mediated by activation of PPG neurons innervating choroid, and in all likelihood those innervating orbital vessels feeding choroid as well. The increase in ChBF with NTS activation that is not blocked by NPA inhibition of NO production may be mediated by VIP. Basal ChBF was not significantly diminished by NPA. It may be that basal ChBF is perhaps more dependent on VIP, and perhaps NO formed by endothelial NOS, than NO produced by PPG nerve terminals (Zagvazdin et al., 1996a,b). It should be noted, however, that in principle NPA could have blocked NO formation at the synapses between terminals of SSN prechoroidal neurons and their target PPG choroidal neurons, since SSN prechoroidal neurons have been observed by us to contain nNOS (Cuthbertson et al., 2003).

Our overall results suggest a role of baroresponsive NTS in the well demonstrated phenomenon of ChBF baroregulation, via its output to choroidal neurons of SSN. The NTS neurons projecting to choroidal SSN and those NTS sites yielding ipsilateral ChBF increases include both the parts of NTS receiving ABP-related cardiovascular afferents and surrounding parts of NTS that show responses to systemic hypotension. The baroresponsive neurons in the NTS territory surrounding baroreceptive NTS may receive their $\mathrm{ABP}$ information by means of input from baroreceptive NTS. It is unclear, however, how low BP signals to NTS from the aortic depressor nerve (ADN), which fires at a low rate during systemic hypotension (Zhang and Mifflin, 2000) could yield increased firing in the output to SSN, and ultimately to choroidal vasodilation. One possibility is that SSNprojecting NTS neurons receive innervation from inhibitory ADN-receptive NTS neurons, the latter of which may reside mainly in baroreceptive NTS and account for the SSN-projecting neurons found outside of baroreceptive NTS. During normal systemic ABP, the SSN-projecting NTS neurons would be inhibited by these ADN-receptive NTS neurons. When BP is low, ADN-receptive NTS neurons would fire at a low rate, with SSNprojecting NTS neurons then disinhibited. Other studies by us have supported this hypothesis (Li et al., 2016).

\section{NTS and Blood Pressure Regulation}

The parts of NTS projecting to the SSN are known to receive $\mathrm{ADN}$ baroreceptive input via the vagus nerve and/or respond to systemic ABP fluctuation (Miura and Reis, 1972; Ciriello, 1983; Rogers et al., 1993; Zhang and Mifflin, 2000; Guyenet, 2006; Li et al., 2010), with increased BP increasing firing in the ADN and its target NTS neurons, and decreased ABP decreasing ADN firing and its target NTS neurons. The baroreceptive part of NTS is involved in regulation of systemic ABP by means of a multisynaptic output to sympathetic preganglionic neurons. In particular, baroreceptive NTS projects to GABAergic neurons of the caudal ventrolateral medulla (CVLM), which in turn project to excitatory neurons of the rostral ventrolateral medulla (RVLM) that project to sympathetic preganglionic neurons of the spinal cord (Schreihofer and Guyenet, 2003). Activation of baroreceptive NTS by high BP thus leads to activation of CVLM via NTS, leading to increased inhibition of RVLM, and diminished excitatory outflow from RVLM to sympathetic preganglionic neurons (Guyenet, 2006). This leads to reduced systemic vasoconstriction that serves to combat the heightened $\mathrm{BP}$, and thereby mediates one part of the systemic baroreflex, together with decreased HR (Guyenet, 2006). Consistent with this, the present results showed reduced ABP with cathodal stimulation of baroresponsive NTS, as did some prior studies with electrical or chemical stimulation of baroreceptive NTS (Crill and Reis, 1968; de Jong et al., 1975; Talman et al., 1984).

\section{Functional and Eye Disease-Related Implications}

The linkages between systemic and ocular circuitries, as in the case of NTS, reinforces the possibility that control of the two operates in parallel but in opposite directions-with the systemic sympathetic control serving to maintain systemic BP in the face of episodic declines in BP, and the parasympathetic control of $\mathrm{ChBF}$ serving to maintain high $\mathrm{ChBF}$ during such bouts of low systemic BP. Since the NTS also exerts a vasodilatory influence on cerebral blood flow (Nakai and Ogino, 1984; Agassandian et al., 2002, 2003), it may be that ocular and cerebral circulations are jointly regulated by the $\mathrm{NTS} \rightarrow \mathrm{SSN} \rightarrow \mathrm{PPG}$ circuit. Consistent with the protective role that cranial parasympathetic vascular regulation might play, severing PPG input to the cerebral vasculature intensifies the cerebral damage occurring with an ischemic event (Kano et al., 1991; Koketsu et al., 1992) and lesioning SSN leads to retinal pathology and functional impairment in rats (Reiner et al., 2010a). It may be that sympathetic innervation of the choroid and sympathetic innervation of peripheral blood vessels also work in opposite directions during ABP fluctuations, with (for example) high ABP leading to reduced sympathetic outflow to the periphery to mitigate the high $\mathrm{ABP}$ but enhanced sympathetic drive to the choroid to prevent overperfusion. Of particular note with regard to the eye, aortic baroreceptors provide the signal to NTS needed for activation of neurogenic choroidal baroregulation (Ciriello, 1983; Donoghue et al., 1985; Rogers et al., 1993), and baroreceptor function shows agerelated decline, and is impaired by smoking, hypertension and diabetes (Brown et al., 1976; Fazan et al., 2001, 2006; do Carmo et al., 2007). The parasympathetic wing of baroregulation that mediates choroidal vasodilation via the NTS $\rightarrow$ SSN $\rightarrow$ PPG circuit during low BP may, thus, be impaired under these circumstances. This could help account for why choroidal baroregulation tends to be impaired by age, smoking, hypertension and diabetes (Movaffaghy et al., 2002; Polak et al., 2003; Wimpissinger et al., 2003; Reiner et al., 2011), and contribute to ChBF defects that harm the retina, and at least in part account for why these are all risk factors for age-related macular degeneration (Hageman et al., 2009).

\section{AUTHOR CONTRIBUTIONS}

CL, MECF, NDM and AR carried out research. CL, MECF and $\mathrm{AR}$ analyzed data. CL, MECF and AR wrote the manuscript. 


\section{FUNDING}

Supported by NIH-EY-05298 and The Methodist Hospitals Endowed Professorship in Neuroscience (AR), the Department of Ophthalmology at UTHSC (MECF) and the University of Tennessee Neuroscience Institute (CL).

\section{REFERENCES}

Agassandian, K., Fazan, V. P., Adanina, V., and Talman, W. T. (2002). Direct projections from the cardiovascular nucleus tractus solitarii to pontine preganglionic parasympathetic neurons: a link to cerebrovascular regulation. J. Comp. Neurol. 452, 242-254. doi: 10.1002/cne.10372

Agassandian, K., Fazan, V. P., Margaryan, N., Dragon, D. N., Riley, J., and Talman, W. T. (2003). A novel central pathway links arterial baroreceptors and pontine parasympathetic neurons in cerebrovascular control. Cell. Mol. Neurobiol. 23, 463-478. doi: 10.1023/A:1025059710382

Alm, A. (1992). "Ocular circulation," in Adler's Physiology of the Eye: Clinical Application, ed. W. M. Hart (St Louis, MO: Mosby), 198-227.

Alm, A., and Bill, A. (1973). Ocular and optic nerve blood flow at normal and increased intraocular pressures in monkeys (Macaca irus): a study with radioactively labelled microspheres including flow determinations in brain and some other tissues. Exp. Eye Res. 15, 15-29. doi: 10.1016/0014-4835(73) 90185-1

Almond, N. E., and Wheatley, A. M. (1992). Measurement of hepatic perfusion in rats by laser Doppler flowmetry. Am. J. Physiol. 262, G203-G209.

Altschuler, S. M., Bao, X. M., Bieger, D., Hopkins, D. A., and Miselis, R. R. (1989). Viscerotopic representation of the upper alimentary tract in the rat: sensory ganglia and nuclei of the solitary and spinal trigeminal tracts. J. Comp. Neurol. 283, 248-268. doi: 10.1002/cne.902830207

Ayajiki, K., Tanaka, T., Okamura, T., and Toda, N. (2000). Evidence for nitroxidergic innervation in monkey ophthalmic arteries in vivo and in vitro. Am. J. Physiol. Heart Circ. Physiol. 279, H2006-H2012.

Bagshaw, E. V., and Evans, M. H. (1976). Measurement of current spread from microelectrodes when stimulating within the nervous system. Exp. Brain Res. 25, 391-400. doi: 10.1007/bf00241729

Bonner, R. F., and Nossal, R. (1990). "Principles of laser-Doppler flowmetry," in Laser-Doppler Blood Flowmetry, eds A. P. Shepherd and P. Öberg (Norwell, MA: Kluwer Academic Publishers), 17-45.

Brown, A. M., Saum, W. R., and Tuley, F. H. (1976). A comparison of aortic baroreceptor discharge in normotensive and spontaneously hypertensive rats. Circ. Res. 39, 488-496. doi: 10.1161/01.res.39.4.488

Chou, P. I., Lu, D. W., and Chen, J. T. (2002). Effect of sympathetic denervation on rabbit choroidal blood flow. Ophthalmologica 216, 60-64. doi: 10. $1159 / 000048299$

Ciriello, J. (1983). Brainstem projections of aortic baroreceptor afferent fibers in the rat. Neurosci. Lett. 36, 37-42. doi: 10.1016/0304-3940(83)90482-2

Collier, R. H. (1967). Experimental embolic ischemia of the choroid. Arch. Ophthalmol. 77, 683-692. doi: 10.1001/archopht.1967.009800206 85025

Cooper, G. R., Mialkowski, K., and Wolff, D. J. (2000). Cellular and enzymatic studies of $\mathrm{N}^{\omega}$-propyl-L-arginine and S-ethyl-N-[4(trifluoromethyl)phenyl]isothiourea as reversible, slowly dissociating inhibitors selective for the neuronal nitric oxide synthase isoform. Arch. Biochem. Biophys. 375, 183-194. doi: 10.1006/abbi.1999.1658

Crill, W. E., and Reis, D. J. (1968). Distribution of carotid sinus and depressor nerves in cat brain stem. Am. J. Physiol. 214, 269-276.

Cuthbertson, S., Jackson, B., Toledo, C., Fitzgerald, M. E., Shih, Y. F., Zagvazdin, Y., et al. (1997). Innervation of orbital and choroidal blood vessels by the pterygopalatine ganglion in pigeons. J. Comp. Neurol. 386, 422-442. doi: 10.1002/(SICI)1096-9861(19970929)386:3<422::AID-CNE7>3.0.CO;2-3

Cuthbertson, S., Ledoux, M. S., Jones, S., Jones, J., Zhou, Q., Gong, S., et al. (2003). Localization of preganglionic neurons that innervate choroidal neurons of pterygopalatine ganglion. Invest. Ophthalmol. Vis. Sci. 44, 3713-3724. doi: 10. 1167/iovs.02-1207

\section{ACKNOWLEDGMENTS}

Special thanks to Dr. Yunping Deng for valuable advice on technical matters, and Aminah Henderson, Marion Joni, Ting Wong, Phoungdinh Nguyen and Amanda Walters for their excellent technical assistances.

de Jong, W., Zandberg, P., and Bohus, B. (1975). Central inhibitory noradrenergic cardiovascular control. Prog. Brain Res. 42, 285-298. doi: 10.1016/s00796123(08)63671-7

Deng, Y. P., Xie, J. P., Wang, H. B., Lei, W. L., Chen, Q., and Reiner, A. (2007). Differential localization of the GluR1 and GluR2 subunits of the AMPA-type glutamate receptor among striatal neuron types in rats. J. Chem. Neuroanat. 33, 167-192. doi: 10.1016/j.jchemneu.2007.02.008

DeoKule, S., Vizzeri, G., Boehm, A. G., Bowd, C., Medeiros, F. A., and Weinreb, R. N. (2009). Correlation among choroidal, parapapillary and retrobulbar vascular parameters in glaucoma. Am. J. Ophthalmol. 147, 736-743.e2. doi: 10.1016/j.ajo.2008.10.020

do Carmo, J. M., Huber, D. A., Castania, J. A., Fazan, V. P. S., Fazan, R. Jr., and Salgado, H. C. (2007). Aortic depressor nerve function examined in diabetic rats by means of two different approaches. J. Neurosci. Methods 161, 17-22. doi: 10.1016/j.jneumeth.2006.10.002

Donoghue, S., Felder, R. B., Gilbey, M. P., Jordan, D., and Spyer, K. M. (1985). Post-synaptic activity evoked in the nucleus tractus solitarius by carotid sinus and aortic nerve afferents in the cat. J. Physiol. 360, 261-273. doi: 10. 1113/jphysiol.1985.sp015616

Dorner, G. T., Garhöfer, G., Huemer, K. H., Golestani, E., Zawinka, C., Schmetterer, L., et al. (2003). Effects of adrenomedullin on ocular hemodynamic parameters in the choroid and the ophthalmic artery. Invest. Ophthalmol. Vis. Sci. 44, 3947-3951. doi: 10.1167/iovs.02-0855

Falsini, B., Anselmi, G. M., Marangoni, D., D’Esposito, F., Fadda, A., Di Renzo, A., et al. (2011). Subfoveal choroidal blood flow and central retinal function in retinitis pigmentosa. Invest. Ophthalmol. Vis. Sci. 52, 1064-1069. doi: 10. 1167/iovs.10-5964

Fazan, V. P. S., Salgado, H. C., and Bareira, A. A. (2001). Aortic depressor nerve unmyelinated fibers in spontaneously hypertensive rats. Am. J. Physiol. Heart Circ. Physiol. 280, H1560-1564.

Fazan, V. P. S., Salgado, H. C., and Bareira, A. A. (2006). Aortic depressor nerve myelinated fibers in acute and chronic experimental diabetes. Am. J. Hypertens. 19, 153-160. doi: 10.1016/j.amjhyper.2005.07.023

Fischer, J. C., Parker, P. M., and Shaw, W. W. (1985). Laser Doppler flowmeter measurements of skin perfusion changes associated with arterial and venous compromise in the cutaneous island flap. Microsurgery 6, 238-243. doi: 10. 1002/micr.1920060410

Fitzgerald, M. E., Gamlin, P. D., Zagvazdin, Y., and Reiner, A. (1996). Central neural circuits for the light-mediated reflexive control of choroidal blood flow in the pigeon eye: a laser Doppler study. Vis. Neurosci. 13, 655-669. doi: 10. $1017 /$ s0952523800008555

Fitzgerald, M. E., Tolley, E., Frase, S., Zagvazdin, Y., Miller, R. F., Hodos, W., et al. (2001). Functional and morphological assessment of age-related changes in the choroid and outer retina in pigeons. Vis. Neurosci. 18, 299-317. doi: 10. $1017 /$ s0952523801182143

Fitzgerald, M. E., Tolley, E., Jackson, B., Zagvazdin, Y. S., Cuthbertson, S. L., Hodos, W., et al. (2005). Anatomical and functional evidence for progressive age-related decline in parasympathetic control of choroidal blood flow in pigeons. Exp. Eye Res. 81, 478-491. doi: 10.1016/j.exer.2005.03.008

Fitzgerald, M. E., Vana, B. A., and Reiner, A. (1990). Control of choroidal blood flow by the nucleus of Edinger-Westphal in pigeons: a laser Doppler study. Invest. Ophthalmol. Vis. Sci. 31, 2483-2492.

Garhöfer, G., Resch, H., Lung, S., Weigert, G., and Schmetterer, L. (2005). Intravenous administration of L-Arginine increases retinal and choroidal blood flow. Am. J. Ophthalmol. 140, 69-76. doi: 10.1016/j.ajo.2005.02.016

Grunwald, J. E., Hariprasad, S. M., DuPont, J., Maguire, M. G., Fine, S. L., Brucker, A. J., et al. (1998a). Foveolar choroidal blood flow in age-related macular degeneration. Invest. Ophthalmol. Vis. Sci. 39, 385-390. 
Grunwald, J. E., Piltz, J., Hariprasad, S. M., and DuPont, J. (1998b). Optic nerve and choroidal circulation in glaucoma. Invest. Ophthalmol. Vis. Sci. 39, 2329-2336.

Grunwald, J. E., Metelitsina, T. I., Dupont, J. C., Ying, G. S., and Maguire, M. G. (2005). Reduced foveolar choroidal blood flow in eyes with increasing AMD severity. Invest. Ophthalmol. Vis. Sci. 46, 1033-1038. doi: 10.1167/iovs.04-1050

Gugleta, K., Orgül, S., Flammer, I., Gherghel, D., and Flammer, J. (2002). Reliability of confocal choroidal laser Doppler flowmetry. Invest. Ophthalmol. Vis. Sci. 43, 723-728.

Guyenet, P. G. (2006). The sympathetic control of blood pressure. Nat. Rev. Neurosci. 7, 335-346. doi: 10.1038/nrn1902

Hageman, G. S. Gehrs, K., Johnson, L. V., and Anderson, D. (2009). Age-related macular degeneration (AMD). Available online at: http://webvision.med.utah.edu/Hagerman.html

Haumschild, D. J. (1986). An overview of laser-Doppler flowmetry. Biomed. Sci. Instrum. 22, 35-40.

Hayreh, S. S. (2004). Posterior ciliary artery circulation in health and disease: the Weisenfeld lecture. Invest. Ophthalmol. Vis. Sci. 45, 749-757. doi: 10. 1167/iovs.03-0469

Housley, G. D., Martin-Body, R. L., Dawson, N. J., and Sinclair, J. D. (1987). Brain stem projections of the glossopharyngeal nerve and its carotid sinus branch in the rat. Neuroscience 22, 237-250. doi: 10.1016/0306-4522(87) 90214-4

Ito, Y. N., Mori, K., Young-Duvall, J., and Yoneya, S. (2001). Aging changes of the choroidal dye filling pattern in indocyanine green angiography of normal subjects. Retina 21, 237-242. doi: 10.1097/00006982-20010600000007

Jablonski, M. M., Iannaccone, A., Reynolds, D. H., Gallaher, P., Allen, S., Wang, X., et al. (2007). Age-related decline in VIP-positive parasympathetic nerve fibers in the human submacular choroid. Invest. Ophthalmol. Vis. Sci. 48, 479-485. doi: 10.1167/iovs.06-0972

Jarajapu, Y. P., Grant, M. B., and Knot, H. J. (2004). Myogenic tone and reactivity of the rat ophthalmic artery. Invest. Ophthalmol. Vis. Sci. 45, 253-259. doi: 10. 1167/iovs.03-0546

Kano, M., Moskowitz, M. A., and Yokota, M. (1991). Parasympathetic denervation of rat pial vessels significantly increases infarction volume following middle cerebral artery occlusion. J. Cereb. Blood Flow Metab. 11, 628-637. doi: 10. 1038/jcbfm.1991.114

Kiel, J. W. (1999). Modulation of choroidal autoregulation in the rabbit. Exp. Eye Res. 69, 413-429. doi: 10.1006/exer.1999.0717

Kiel, J. W., and Shepherd, A. P. (1992). Autoregulation of choroidal blood flow in the rabbit. Invest. Ophthalmol. Vis. Sci. 33, 2399-2410.

Koketsu, N., Moskowitz, M. A., Kontos, H. A., Yokota, M., and Shimizu, T. (1992). Chronic parasympathetic sectioning decreases regional cerebral blood flow during hemorrhagic hypotension and increases infarct size after middle cerebral artery occlusion in spontaneously hypertensive rats. J. Cereb. Blood Flow Metab. 12, 613-620. doi: 10.1038/jcbfm. 1992.85

Li, C., Fitzgerald, M. E. C., Del Mar, N., Cuthbertson, S., LeDoux, M. S., Gong, S., et al. (2015). The identification and neurochemical characterization of central neurons that target parasympathetic preganglionic neurons involved in the regulation of choroidal blood flow in the rat eye using pseudorabies virus, immunolabeling and conventional pathway tracing methods. Front. Neuroanat. 9:65. doi: 10.3389/fnana.2015.00065

Li, C., Fitzgerald, M. E., Ledoux, M. S., Gong, S., Ryan, P., Del Mar, N., et al. (2010). Projections from the hypothalamic paraventricular nucleus and the nucleus of the solitary tract to prechoroidal neurons in the superior salivatory nucleus: pathways controlling rodent choroidal blood flow. Brain Res. 1358, 123-139. doi: 10.1016/j.brainres.2010.08.065

Li, C., Fitzgerald, M. E. C., Del Mar, N., and Reiner, A. (2016). Disinhibition of neurons of the nucleus of solitary tract that project to the superior salivatory nucleus causes choroidal vasodilation: implications for mechanisms underlying choroidal baroregulation. Neurosci. Lett. 633, 106-111. doi: 10. 1016/j.neulet.2016.09.029

Loeffler, K. U., Hayreh, S. S., and Tso, M. O. M. (1994). The effects of simultaneous occlusion of the posterior ciliary artery and vortex veins. a histopathological study. Arch. Ophthalmol. 112, 674-682. doi: 10. 1001/archopht.1994.01090170118033
Loewy, A. D. (1990). "Central autonomic pathways," in Central Regulation of Autonomic Functions, eds A. D. Loewy and K. M. Spyer (New York, NY: Oxford University Press), 88-103.

Lovasik, J. V., Kergoat, H., Riva, C. E., Petrig, B. L., and Geiser, M. (2003). Choroidal blood flow during exercise-induced changes in the ocular perfusion pressure. Invest. Ophthalmol. Vis. Sci. 44, 2126-2132. doi: 10.1167/iovs. 02-0825

Lu, H., Chestek, C. A., Shaw, K. M., and Chiel, H. J. (2008). Selective extracellular stimulation of individual neurons in ganglia. J. Neural Eng. 5, 287-309. doi: 10. 1088/1741-2560/5/3/003

Mayne, R. G., Armstrong, W. E., Crowley, W. R., and Bealer, S. L. (1998). Cytoarchitectonic analysis of Fos-immunoreactivity in brainstem neurones following visceral stimuli in conscious rats. J. Neuroendocrinol. 10, 839-847. doi: 10.1046/j.1365-2826.1998.00271.x

Merrill, D. M., Bikson, M., and Jefferys, J. G. R. (2005). Electrical stimulation of excitable tissue: design of efficacious and safe protocols. J. Neurosci. Methods 141, 171-198. doi: 10.1016/j.jneumeth.2004.10.020

Metelitsina, T. I., Grunwald, J. E., DuPont, J. C., Ying, G. S., Brucker, A. J. and Dunaief, J. L. (2008). Foveolar choroidal circulation and choroidal neovascularization in age-related macular degeneration. Invest. Ophthalmol. Vis. Sci. 49, 358-363. doi: 10.1167/iovs.07-0526

Miura, M., and Reis, D. J. (1972). The role of the solitary and paramedian reticular nuclei in mediating cardiovascular reflex responses from carotid baro- and chemoreceptors. J. Physiol. 223, 525-548. doi: 10.1113/jphysiol.1972.sp009861

Morrison, J. C., Johnson, E. C., Cepurna, W. O., and Funk, R. H. (1999). Microvasculature of the rat optic nerve head. Invest. Ophthalmol. Vis. Sci. 40, 1702-1709.

Movaffaghy, A., Chamot, S. R., Dosso, A., Pournaras, C. J., Sommerhalder, J. R., and Riva, C. E. (2002). Effect of isometric exercise on choroidal blood flow in type I diabetic patients. Klin. Monbl. Augenheilkd. 219, 299-301. doi: 10.1055/s2002-30665

Nakai, M., and Ogino, K. (1984). The relevance of cardio-pulmonary-vascular reflex to regulation of the brain vessels. Jpn. J. Physiol. 34, 193-197. doi: 10. 2170/jjphysiol.34.193

Nilsson, S. F. E. (1996). Nitric oxide as a mediator of parasympathetic vasodilation in ocular and extraocular tissues in the rabbit. Invest. Ophthalmol. Vis. Sci. 37, 2110-2119.

Nilsson, G. E., Tenland, T., and Oberg, P. A. (1980). Evaluation of a laser Doppler flowmeter for measurement of tissue blood flow. IEEE Trans. Biomed. Eng. 27, 597-604. doi: 10.1109/tbme.1980.326582

Novais, E. A., Badaró, E., Allemann, N., Morales, M. S., Rodrigues, E. B., de Souza Lima, R., et al. (2015). Correlation between choroidal thickness and ciliary artery blood flow velocity in normal subjects. Ophthalmic Surg. Lasers Imaging Retina 46, 920-924. doi: 10.3928/23258160-20151008-04

Overend, J., and Martin, W. (2007). Differential effects of nitric oxide synthase inhibitors on endothelium-dependent and nitrergic nerve-mediated vasodilatation in the bovine ciliary artery. Br. J. Pharmacol. 145, 1001-1008. doi: 10.1038/sj.bjp.0707113

Overend, J., Wilson, W. S., and Martin, W. (2005). Biphasic neurogenic vasodilatation in the bovine intraocular long posterior ciliary artery: involvement of nitric oxide and an additional unidentified neurotransmitter. Br. J. Pharmacol. 150, 488-493. doi: 10.1038/sj.bjp.0706264

Paton, J. F. (1999). The Sharpey-Schafer prize lecture: nucleus tractus solitarii: integrating structures. Exp. Physiol. 84, 815-833. doi: 10.1111/j.1469-445X. 1999.01912.x

Paxinos, G., and Watson, C. (2009). The Rat Brain in Stereotaxic Coordinates. 6th edn. New York, NY: Academic Press

Pemp, B., Garhofer, G., Lasta, M., Schmidl, D., Wolzt, M., and Schmetterer, L. (2012). The effects of moxaverine on ocular blood flow in patients with agerelated macular degeneration or primary open angle glaucoma and in healthy control subjects. Acta Ophthalmol. 90, 139-145. doi: 10.1111/j.1755-3768.2010. 01878.x

Pilowsky, P. M., and Goodchild, A. K. (2002). Baroreceptor reflex pathways and neurotransmitters: 10 years on. J. Hypertens. 20, 1675-1688. doi: 10. 1097/00004872-200209000-00002

Polak, K., Polska, E., Luksch, A., Dorner, G., Fuchsjäger-Mayrl, G., Findl, O., et al. (2003). Choroidal blood flow and arterial blood pressure. Eye (Lond) 17, 84-88. doi: $10.1038 /$ sj.eye. 6700246 
Pournaras, C. J., Logean, E., Riva, C. E., Petrig, B. L., Chamot, S. R., Coscas, G., et al. (2006). Regulation of subfoveal choroidal blood flow in age-related macular degeneration. Invest. Ophthalmol. Vis. Sci. 47, 1581-1586. doi: 10.1167/iovs.050434

Ranck, J. B. Jr. (1975). Which elements are excited in electrical stimulation of mammalian central nervous system: a review. Brain Res 98, 417-440. doi: 10. 1016/0006-8993(75)90364-9

Rechtman, E., Harris, A., Seisky, B., Kagemann, L., Danis, R. P., Sines, D., et al. (2007). The relationship between retrobulbar and choroidal hemodynamics in non-neovascular age-related macular degeneration. Ophthalmic Surg. Lasers Imaging 38, 219-225.

Reiner, A., Del Mar, N., Zagvazdin, Y., Li, C., and Fitzgerald, M. E. (2011). Agerelated impairment in choroidal blood flow compensation for arterial blood pressure fluctuation in pigeons. Invest. Ophthalmol. Vis. Sci. 52, 7238-7247. doi: 10.1167/iovs.10-6464

Reiner, A., Fitzgerald, M. E., and Li, C. (2012). "Neural control of ocular blood flow," in Ocular Blood Flow, eds L. Schmetterer and J. W. Kiel (Berlin: Springer), 243-309.

Reiner, A., Fitzgerald, M. E. C., Li, C., and Del Mar, N. (2010a). Effects on rat retina of disrupted parasympathetic choroidal blood flow regulation by superior salivatory nucleus lesions. 51, ARVO Abst. \# 6323.

Reiner, A., Li, C., Del Mar, N., and Fitzgerald, M. E. (2010b). Choroidal blood flow compensation in rats for arterial blood pressure decreases is neuronal nitric oxide-dependent but compensation for arterial blood pressure increases is not. Exp. Eye Res. 90, 734-741. doi: 10.1016/j.exer.2010. 03.006

Reiner, A., Zagvazdin, Y., and Fitzgerald, M. E. (2003). Choroidal blood flow in pigeons compensates for decreases in arterial blood pressure. Exp. Eye Res. 76, 273-282. doi: 10.1016/s0014-4835(02)00316-0

Riva, C. E., Cranstoun, S. D., Mann, R. M., and Barnes, G. E. (1994). Local choroidal blood flow in the cat by laser Doppler flowmetry. Invest. Ophthalmol. Vis. Sci. 35, 608-618.

Rogers, R. F., Paton, J. F., and Schwaber, J. S. (1993). NTS neuronal responses to arterial pressure and pressure changes in the rat. Am. J. Physiol. 265, R1355-1368.

Schreihofer, A. M., and Guyenet, P. G. (2003). Baro-activated neurons with pulsemodulated activity in the rat caudal ventrolateral medulla express GAD67 mRNA. J. Neurophysiol. 89, 1265-1277. doi: 10.1152/jn.00737.2002

Shepherd, A. P., Riedel, G. L., Kiel, J. W., Haumschild, D. J., and Maxwell, L. C. (1997). Evaluation of an infrared laser-Doppler blood flowmeter. Am. J. Physiol. 252, G832-G839.

Steinle, J. J., Krizsan-Agbas, D., and Smith, P. G. (2000). Regional regulation of choroidal blood flow by autonomic innervation in the rat. Am. J. Physiol. Regul. Integr. Comp. Physiol. 279, R202-R209.

Stone, R. A., Kuwayama, Y., and Laties, A. M. (1987). Regulatory peptides in the eye. Experientia 43, 791-800. doi: 10.1007/bf01945357

Talman, W. T., Granata, A. R., and Reis, D. J. (1984). Glutamatergic mechanisms in the nucleus tractus solitarius in blood pressure control. Fed. Proc. 43, 39-44.
Toda, N., Toda, M., Ayajiki, K., and Okamura, T. (1998). Cholinergic nerve function in monkey ciliary arteries innervated by nitroxidergic nerve. Am. J. Physiol. 274, H1582-H1589.

Tonini, M., Khayi, H., Pepin, J. L., Renard, E., Baguet, J. P., Lévy, P., et al. (2010). Choroidal blood-flow responses to hyperoxia and hpercapnia in men with obstructive sleep apnea. Sleep 33, 811-818.

Wiencke, A. K., Nilson, H., Nielsen, P. J., and Nyborg, N. C. B. (1994). Nonadrenergic noncholinergic vasodilation in bovine ciliary artery involves CGRP and neurogenic nitric oxide. Invest. Ophthalmol. Vis. Sci. 35, 3268-3277.

Wimpissinger, B., Resch, H., Berisha, F., Weigert, G., Polak, K., and Schmetterer, L. (2003). Effects of isometric exercise on subfoveal choroidal blood flow in smokers and nonsmokers. Invest. Ophthalmol. Vis. Sci. 44, 4859-4863. doi: 10. 1167/iovs.03-0391

Xu, W., Grunwald, J. E., Metelitsina, T. I., DuPont, J. C., Ying, G. S., Martin, E. R., et al. (2010). Association of risk factors for choroidal neovascularization in agerelated macular degeneration with decreased foveolar choroidal circulation. Am. J. Ophthalmol. 150, 40.e2-47.e2. doi: 10.1016/j.ajo.2010.01.041

Zagvazdin, Y. S., Fitzgerald, M. E., Sancesario, G., and Reiner, A. (1996a). Neural nitric oxide mediates Edinger-Westphal nucleus evoked increase in choroidal blood flow in the pigeon. Invest. Ophthalmol. Vis. Sci. 37, 666-672.

Zagvazdin, Y. S., Sancesario, G., Wang, Y. X., Share, L., Fitzgerald, M. E., and Reiner, A. (1996b). Evidence from its cardiovascular effects that 7-nitroindazole may inhibit endothelial nitric oxide synthase in vivo. Eur. J. Pharmacol. 303, 61-69. doi: 10.1016/0014-2999(96)00106-9

Zhang, L. L., and Ashwell, K. W. (2001). Development of the cyto-and chemoarchitectural organization of the rat nucleus of the solitary tract. Anat. Embryol. (Berl) 203, 265-282. doi: 10.1007/s004290000151

Zhang, H. Q., Fast, W., Marletta, M. A., Martasek, P., and Silverman, R. B. (1997). Potent and selective inhibition of neuronal nitric oxide synthase by $\mathrm{N}$ omegapropyl-L-arginine. J. Med. Chem. 40, 3869-3870. doi: 10.1021/jm970550g

Zhang, J., and Mifflin, S. W. (2000). Responses of aortic depressor nerve-evoked neurones in rat nucleus of the solitary tract to changes in blood pressure. J. Physiol. 529, 431-443. doi: 10.1111/j.1469-7793.2000.00431.x

Zion, I. B., Harris, A., Siesky, B., Shulman, S., McCranor, L., and Garzozi, H. J. (2007). Pulsatile ocular blood flow: relationship with flow velocities in vessels supplying the retina and choroid. Br. J. Ophthalmol. 91, 882-884. doi: 10 . 1136/bjo.2006.108340

Conflict of Interest Statement: The authors declare that the research was conducted in the absence of any commercial or financial relationships that could be construed as a potential conflict of interest.

Copyright (C) 2016 Li, Fitzgerald, Del Mar and Reiner. This is an open-access article distributed under the terms of the Creative Commons Attribution License (CC BY). The use, distribution and reproduction in other forums is permitted, provided the original author(s) or licensor are credited and that the original publication in this journal is cited, in accordance with accepted academic practice. No use, distribution or reproduction is permitted which does not comply with these terms. 